\title{
Measurement-based Optimization of Batch and Repetitive Processes using an Integrated Two-Layer Architecture
}

\author{
M. Podmajerský ${ }^{\mathrm{a}}$, M. Fikar ${ }^{\mathrm{a}}$, and B. Chachuat ${ }^{\mathrm{b}, 1}$ \\ ${ }^{\mathrm{a}}$ Institute of Information Engineering, Automation and Mathematics, STU in \\ Bratislava, Slovakia \\ ${ }^{\mathrm{b}}$ Centre for Process System Engineering, Department of Chemical Engineering, \\ Imperial College London, SW7 2AZ, UK
}

\begin{abstract}
This paper is concerned with optimal control of batch and repetitive processes in the presence of uncertainty. An integrated two-layer optimization strategy is proposed, whereby within-run corrections are performed using a neighboring-extremal update strategy and run-to-run corrections are based on a constraint-adaptation scheme. The latter is appealing since a feasible operating strategy is guaranteed upon convergence, and its combination with neighboring-extremal updates improves the reactivity and convergence speed. Moreover, these two layers are consistent in that they share the same objective function. The proposed optimization scheme is declined into two versions, namely an indirect version based on the Pontryagin maximum principle and a direct version that applies a control parameterization and nonlinear programming techniques. Although less rigorous, the latter approach can deal with singular extremals and path constraints as well as handle active-set changes more conveniently. Two case studies are considered. The indirect approach is demonstrated for a level-control problem in an experimental two-tank system, whereas the direct approach is illustrated in numerical simulation on a fed-batch reactor for acetoacetylation of pyrrole. The results confirm that faster adaptation is possible with the proposed integrated two-layer scheme compared to either constraint adaptation or neighboring-extremal update alone.
\end{abstract}

Keywords: batch processes; repetitive processes; dynamic optimization; optimal control; neighboring-extremal updates; run-to-run optimization; constraint adaptation; two-layer architecture

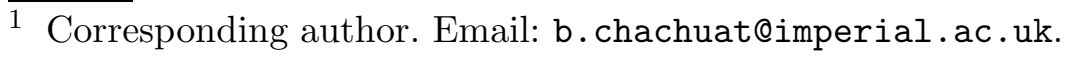




\section{Introduction}

Industrial chemical processes can be subject to large uncertainty during their operation. Common sources of uncertainty include measurement noise, incomplete knowledge of a reaction scheme, uncertain kinetic parameters, inaccurate heat or mass transfer coefficients, feed impurities, and fouling. This usually entails a lower production quantity or product quality, along with possible operational or safety constraint violations. Optimal control has the ability to mitigate the effect of uncertainty on process performance, especially in the presence of constraints and complex dynamics [1].

An increasing number of approaches for dealing with uncertainty and disturbances in dynamic processes can be found in the literature. Traditional nonlinear model predictive control (NMPC) [2-4], and more recently MPC with an economic objective - also known as dynamic real-time optimization (DRTO) - $[1,5,6]$, implement a reoptimization strategy and use measurements to update the current initial states and/or parameters in the model. A brute force reoptimization approach suffers important limitation though, as the required computations may not be tractable fast enough in real-time or may need expensive hardware and software setup; see, e.g., [6] and reference therein. Clearly, the time needed to optimize a system depends both on the problem complexity and on the available computational power. In this regard, an acceptable compromise is not always possible in practice, since long delays between the reoptimizations may as well lead to dramatic performance loss or, worse, to constraint violation and instability. This is especially true for chemical processes exhibiting fast nonlinear dynamics. In response to this, advanced NMPC and DRTO techniques relying on fast update strategies based on sensitivity information have been developed in recent years [7-10]. Perhaps the biggest difficulty with such multi-layered schemes, however, is defining optimization subproblems that remain consistent across the different layers, similar to the challenges encountered with classical real-time optimization [11].

Another technique is the so-called explicit MPC approach [12-16], which uses multi-parametric programming to compute, in an off-line manner, all possible control actions for a given range of the state variables. Then, the control inputs are simply adjusted by selecting the control law that corresponds to the actual state of the process, as given by the latest measurements or estimates. Despite its ability to accommodate fast sampling times, perhaps the foremost limitation of this approach comes from the curse of dimensionality. This currently limits applications of explicit MPC to problems having no more than a few state variables as well as linear or piecewise-linear dynamics, although efficient model-order reduction and approximation techniques can alleviate the problem dramatically [17]. The NCO-tracking methodology [18-20] also aims to get rid of the online computational burden by transforming a (dynamic) 
optimization problem into a feedback control problem, which is robust towards uncertainty. This transformation typically requires approximations and makes the assumption that the structure and active constraints in the optimal solution are invariant in the presence of uncertainty.

This paper presents a two-layer decomposition approach tailored to batch and repetitive process optimization [21]. The outer layer applies run-to-run corrections via the adaptation of the terminal constraints [22], and it is integrated with an inner layer that performs within-run corrections based on neighboring-extremal (NE) updates [23, 24]. This scheme is similar in essence to the two-layer optimization schemes in [10, 25, 26], where NMPC or DRTO is implemented at the slow time scale and a quick sensitivity update is performed at the fast time scale to reduce the optimality loss and promote stability.

In the outer layer, it is the terminal constraints that are adapted between two runs, based on the mismatch between their predicted and measured values, instead of adapting the initial conditions as in a receding-horizon strategy. Although typically suboptimal, this adaptation approach alleviates the need for reestimating (part of) the model parameters between the runs and a feasible operation is guaranteed upon convergence. Optimal operation can even be ascertained when the optimum is completely determined by constraints; see [22]. The inner layer features fast, sensitivity-based updates in an objective to reject within-run disturbances, enforce feasibility, and promote optimality. NE theory, which has been developed over the last 4-5 decades to avoid the full and costly reoptimization of nonlinear systems, is well-suited for this purpose. This combination makes the proposed two-layer scheme consistent in the sense that both layers share the same objective function as the NE updates are computed from a linearization of the (adapted) dynamic optimization problem.

State-of-the-art numerical techniques for solving optimal control problems can be classified as direct methods, whereby the control trajectories are parameterized and an approximate optimal solution is computed by solving the resulting finite-dimensional optimization problem (NLP) [27, 28]; and indirect methods, where one seeks for trajectories that satisfy the Pontryagin maximum principle, without the need to resort to a control parameterization [29, 30]. Accordingly, NE updates can be computed from the linearization of either the parameterized optimal control problem, using NLP sensitivity theory [24], or of the original optimal control problem, using NE control theory [23]. It follows that the proposed integrated two-layer architecture itself can be declined into two versions, namely a direct scheme and an indirect scheme. Although less rigorous than the indirect approach due to the need for a parameterization, the direct scheme can deal with singular extremals and path constraints more conveniently and it can also handle active-set changes.

The remainder of the paper is organized as follows. The optimization problem 
formulation as well as background elements on run-to-run optimization using constraint adaptation are presented in Sect. 2. A high-level description of the integrated two-layer optimization scheme is given in Sect. 3, before detailing the indirect and direct variants in Sect. 4 and Sect. 6, respectively. The former is demonstrated for a level-control problem in an experimental two-tank system in Sect. 5, whereas the later is illustrated in numerical simulation on a fed-batch reactor for acetoacetylation of pyrrole in Sect. 7. Finally, Sect. 8 concludes the paper.

\section{Run-to-Run Optimization}

\subsection{Dynamic Optimization Formulation}

The following dynamic optimization problem with terminal constraints is considered:

$$
\begin{array}{cl}
\min _{\boldsymbol{u}} & \phi\left(\boldsymbol{x}\left(t_{\mathrm{f}}\right)\right)+\int_{0}^{t_{\mathrm{f}}} L(\boldsymbol{x}(t), \boldsymbol{u}(t)) \mathrm{d} t \\
\text { s.t. } & \dot{\boldsymbol{x}}(t)=\boldsymbol{F}(\boldsymbol{x}(t), \boldsymbol{u}(t)), \quad 0 \leq t \leq t_{\mathrm{f}} \\
& \boldsymbol{x}(0)=\boldsymbol{x}_{0} \\
& \boldsymbol{u}^{L} \leq \boldsymbol{u}(t) \leq \boldsymbol{u}^{U} \\
& \boldsymbol{\psi}\left(\boldsymbol{x}\left(t_{\mathrm{f}}\right)\right) \leq \mathbf{0} .
\end{array}
$$

In (1)-(5), $t \geq 0$ denotes the independent time variable, with $t_{\mathrm{f}}$ the final time; $\boldsymbol{u}(t) \in \mathbb{R}^{n_{u}}$ the control vector; $\boldsymbol{x}(t) \in \mathbb{R}^{n_{x}}$ the state vector, with initial value $\boldsymbol{x}_{0} ; \phi$ and $L$ the terminal cost and the integral cost, respectively; and $\boldsymbol{\psi}$ the vector of $n_{\psi}$ terminal constraints. All the functions in (1)-(5) are assumed to be twice continuously differentiable in all their arguments.

\subsection{Run-to-Run Constraint Adaptation}

The principle behind run-to-run (or batch-to-batch) optimization is similar to NMPC or DRTO with regards to the fact that the optimization model is adapted prior to rerunning the optimizer - e.g., initial state or model parameter update. An important difference nonetheless is that the control horizon is not shifted or reduced. In run-to-run constraint adaptation [22], more specifically, it is the terminal constraints (5) in the optimization model that are adapted after each run as follows:

$$
\boldsymbol{\psi}\left(\boldsymbol{x}\left(t_{\mathrm{f}}\right)\right) \leq \delta \boldsymbol{\psi}_{k}
$$


where $\delta \psi_{k}$ stands for the terminal constraint bias in the $k$ th run.

The run-to-run constraint-adaptation scheme is depicted in Fig. 1. The constrained dynamic optimization problem relies on the nominal process model, which is left unchanged, while the adaptation proceeds by substituting (5) with (6). This problem is reoptimized prior to starting the next run, using either a direct or an indirect numerical solution procedure. The computed optimal control trajectory $\boldsymbol{u}_{k}^{*}(t), 0 \leq t \leq t_{\mathrm{f}}$, is then applied to the plant during the $k$ th run. At the end of the $k$ th run, the discrepancy between the measured terminal constraint values, $\boldsymbol{\psi}_{k}^{\text {meas }}$, and the optimizer predictions $\boldsymbol{\psi}\left(\boldsymbol{x}_{k}^{*}\left(t_{\mathrm{f}}\right)\right)$, where $\boldsymbol{x}_{k}^{*}(t)$ denotes the predicted optimal response, is then used to update the constraint bias $\delta \boldsymbol{\psi}_{k+1}$. In turn, the optimizer is rerun, and so forth.

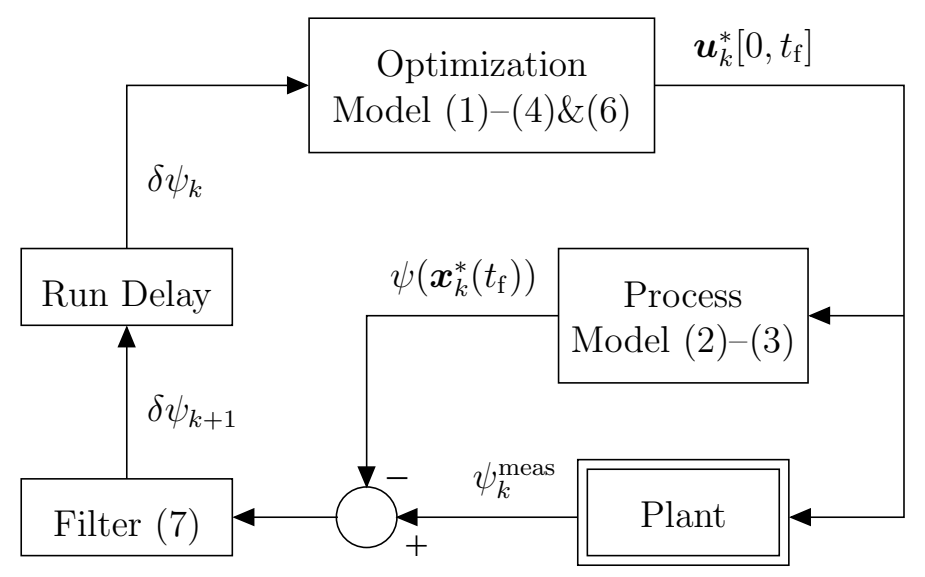

Fig. 1. Optimization scheme that uses run-to run constraint adaptation.

The constraint bias in run $k$ can be directly updated as the difference between the available terminal constraint measurements $\boldsymbol{\psi}_{k}^{\text {meas }}$ and the predicted constraint values $\boldsymbol{\psi}\left(\boldsymbol{x}_{k}^{*}\left(t_{\mathrm{f}}\right)\right)$. Such a simple strategy can lead to over-correcting the constraints, for instance when operating far away from the constraint limits, and it can also exacerbate the sensitivity of the adaptation scheme to measurement noise. Therefore, a more practical strategy involes filtering the bias, e.g., with a first-order exponential filter:

$$
\delta \boldsymbol{\psi}_{k+1}=\left[\boldsymbol{I}-\boldsymbol{W}_{\psi}\right] \delta \boldsymbol{\psi}_{k}+\boldsymbol{W}_{\psi}\left[\boldsymbol{\psi}_{k}^{\text {meas }}-\boldsymbol{\psi}\left(\boldsymbol{x}_{k}\left(t_{\mathrm{f}}\right)\right)\right]
$$

with $k$ the run index, and $\boldsymbol{W}_{\psi}$ a gain matrix - typically, a diagonal matrix with entries $w_{\psi_{i}}$ such that $0<w_{\psi_{i}} \leq 1$, for each $i=1, \ldots, n_{\psi}$; compare also [31, 32].

It is noteworthy that this approach can be readily applied to problems with state path constraints as well, for instance by discretizing the path constraints into interior point constraints. Another approach, described in [22], involves defining time-varying biases that correspond to the difference between the actual and predicted values of the state path constraints during the entire run. 


\section{Integrated Two-Layer Architecture}

Run-to-run constraint adaptation is appealing as it provides a natural framework to handle changes in active constraints, both path and terminal constraints, and it can guarantee feasibility upon convergence. Moreover, it is quite robust towards model mismatch and process disturbances, and since it does not rely on a model parameter update its practical implementation is rather simple. Nonetheless, an inherent limitation of run-to-run adaptation is the absence of control corrections during the runs, and so within-run disturbances cannot be rejected. Run-to-run adaptation also lacks reactivity in that the convergence to a feasible operation can be rather slow, and failure to adapt the objective and constraint gradients typically leads to suboptimal operation $[22,32]$.

On the other hand, NE updates are so designed to cancel out the first variation of the necessary conditions for optimality, without the need for a full reoptimization. As such, they are particularly efficient at correcting small deviations around a nominal extremal path in order to deliver near-optimal performance cheaply. Nonetheless, the performance of NE updates can decrease dramatically in the presence of large model mismatch and process disturbances. In particular, NE updates do not come along with any feasibility guarantee.

Our proposal in this paper is to combine run-to-run constraint adaptation with NE updates in a two-layer architecture, in an attempt to complement their respective strengths and mitigate their deficiencies. This integrated two-layer optimization scheme is depicted in Fig. 2, and proceeds as follows:

- Outer Layer - Run-to-run constraint adaptation is applied in order to handle large model mismatch and changes in active constraints. This layer relies on run-end measurements only, similar to the description in Sect. 2.2.

- Inner Layer - NE updates use within-run measurement information in order to enhance the speed of convergence of the integrated scheme by reducing the feasibility gap. These updates also have a control action by rejecting within-run disturbances in a near-optimal manner.

Because both layers share the same objective function, this two-layer decomposition is consistent. Consistency is especially important in order to mitigate the loss of performance, as discussed for instance in [5] and [26].

Notwithstanding its attractive features, a current limitation of this two-layer architecture is the need for a full-state measurement to compute the NE updates, which is seldom available in practice. This calls for the use of on-line state estimation techniques in the inner layer, such as an extended Kalman filter and its unscented variant [33] or a moving horizon estimator [34]; this on-line estimation block would be placed right after the 'Plant' block in Fig. 2, 


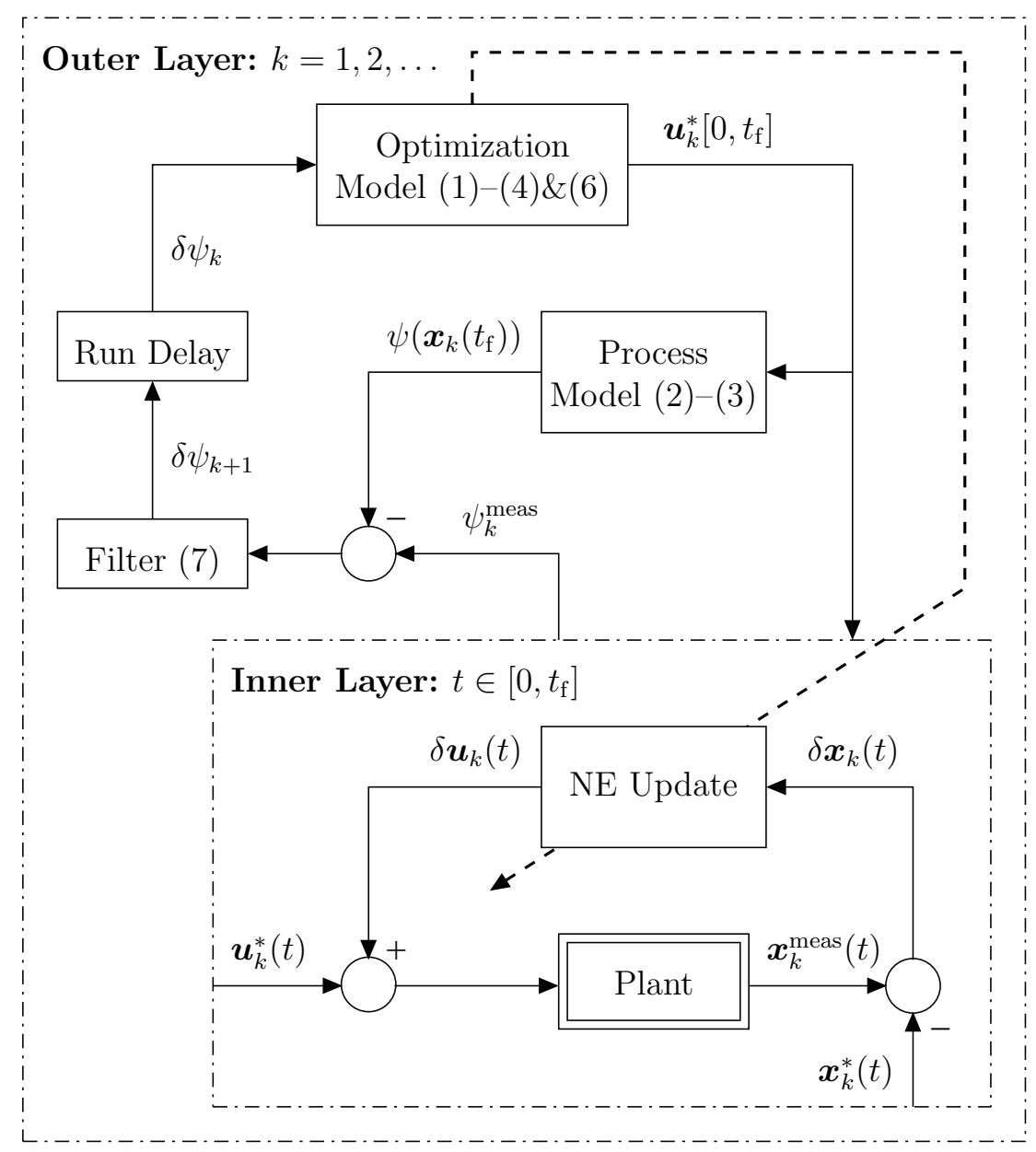

Fig. 2. Two-layer optimization scheme that uses run-to run constraint adaptation in the outer layer and NE updates in the inner layer.

taking measured output variables $\boldsymbol{y}_{k}^{\text {meas }}$ as inputs and outputting estimated state variables $\boldsymbol{x}_{k}^{\text {est }}$ to be used in the 'NE Update' block. Also note that this limitation is not at all specific to the proposed optimization scheme, but it is a shared issue with other model-based control and real-time optimization architectures; see, e.g., [26, 35, 36].

The proposed two-layer architecture comes in two versions, namely a direct scheme and an indirect scheme. In the indirect approach, both the solution to the optimal control problem and the derivation of the NE update scheme are based on the Pontryagin maximum principle. In contrast, the direct approach first approximates the optimal control problem as an NLP and relies on this approximation to compute the NE updates. Although less rigorous, the direct approach proves easier to devise, especially for singular control problems or in the presence of state path constraints. Moreover, the loss of performance associated with the parameterization is seldom noticed when a tailored control parameterization is used. Both the indirect and direct approaches are described, illustrated and discussed in the remainder of the paper. 


\section{Indirect Two-Layer Scheme}

Indirect methods for optimal control seek trajectories that satisfy the Pontryagin maximum principle, without the need to resort to a control parameterization. This section begins by reviewing the relevant optimality conditions as well as NE theory and its practical implementation, before stating the indirect two-layer optimization algorithm.

Necessary Conditions for Optimality We assume that the optimal control problem is not abnormal and that the terminal constraints satisfy a constraint qualification. Following [23] and in order to keep the notation simple, we consider the Hamiltonian function $\mathcal{H}$ as well as the generalized terminal term $\Phi$ defined as:

$$
\begin{aligned}
\mathcal{H}\left(\boldsymbol{x}, \boldsymbol{u}, \boldsymbol{\lambda}, \boldsymbol{\mu}^{L}, \boldsymbol{\mu}^{U}\right) & :=L(\boldsymbol{x}, \boldsymbol{u})+\boldsymbol{F}(\boldsymbol{x}, \boldsymbol{u})^{\top} \boldsymbol{\lambda}+\boldsymbol{\mu}^{L^{\top}}\left(\boldsymbol{u}^{L}-\boldsymbol{u}\right)+\boldsymbol{\mu}^{U^{\top}}\left(\boldsymbol{u}-\boldsymbol{u}^{U}\right) \\
\Phi(\boldsymbol{x}, \overline{\boldsymbol{\nu}}) & :=\phi(\boldsymbol{x})+\overline{\boldsymbol{\nu}}^{\top} \overline{\boldsymbol{\psi}}(\boldsymbol{x})
\end{aligned}
$$

Now, if $\boldsymbol{u}_{k}^{*}$ is an optimal solution to the modified optimal control problem (1)(4)\&(6) in run $k$, then there exist a response trajectory $\boldsymbol{x}_{k}^{*}$, adjoint trajectory $\boldsymbol{\lambda}_{k}^{*}$, multiplier trajectories $\boldsymbol{\mu}_{k}^{L *}$ and $\boldsymbol{\mu}_{k}^{U *}$, and multiplier vector $\boldsymbol{\nu}_{k}^{*}$ such that the following first-order necessary conditions for optimality (NCOs) hold [37]: ${ }^{2}$

$$
\left.\begin{array}{rl}
\dot{\boldsymbol{x}}_{k}^{*}(t) & =\boldsymbol{F}\left(\boldsymbol{x}_{k}^{*}(t), \boldsymbol{u}_{k}^{*}(t)\right) \\
\dot{\boldsymbol{\lambda}}_{k}^{*}(t) & =-\mathcal{H}_{\boldsymbol{x}}\left(\boldsymbol{x}_{k}^{*}(t), \boldsymbol{u}_{k}^{*}(t), \boldsymbol{\lambda}_{k}^{*}(t), \boldsymbol{\mu}_{k}^{L *}(t), \boldsymbol{\mu}_{k}^{U *}(t)\right) \\
\mathbf{0} & =\mathcal{H}_{\boldsymbol{u}}\left(\boldsymbol{x}_{k}^{*}(t), \boldsymbol{u}_{k}^{*}(t), \boldsymbol{\lambda}_{k}^{*}(t), \boldsymbol{\mu}_{k}^{L *}(t), \boldsymbol{\mu}_{k}^{U *}(t)\right) \\
\mathbf{0} & =\boldsymbol{\mu}_{k}^{L *}(t)^{\mathrm{\top}}\left[\boldsymbol{u}^{L}-\boldsymbol{u}_{k}^{*}(t)\right], \quad \boldsymbol{\mu}_{k}^{L *}(t) \geq \mathbf{0}, \quad \boldsymbol{u}_{k}^{*}(t) \geq \boldsymbol{u}^{L} \\
\mathbf{0} & =\boldsymbol{\mu}_{k}^{U *}(t)^{\mathrm{\top}}\left[\boldsymbol{u}_{k}^{*}(t)-\boldsymbol{u}^{U}\right], \quad \boldsymbol{\mu}_{k}^{U *}(t) \geq \mathbf{0}, \quad \boldsymbol{u}_{k}^{*}(t) \leq \boldsymbol{u}^{U}
\end{array}\right\}
$$

where all equalities and inequalities are understood component-wise. The last two conditions in (8) determine the set of active terminal constraints in the optimal solution, denoted by the vector $\overline{\boldsymbol{\psi}}$ of dimension $n_{\bar{\psi}}$ hereafter, along with the corresponding multipliers $\overline{\boldsymbol{\nu}}_{k}^{*}$; inactive constraints, on the other hand, have their corresponding multipliers equal to zero.

$\overline{2}$ Subscript notations such as $y$ for a given function denote partial derivatives of that function with respect to $y$. 
Subsequently, a superscript ${ }^{*}$ indicates that the corresponding quantity is evaluated along an extremal path $\boldsymbol{u}_{k}^{*}$, corresponding states $\boldsymbol{x}_{k}^{*}$, adjoints $\boldsymbol{\lambda}_{k}^{*}$, and multipliers $\boldsymbol{\mu}_{k}^{L *}, \boldsymbol{\mu}_{k}^{U *}$ and $\boldsymbol{\nu}_{k}^{*}$.

Neighboring-Extremal Control Small changes $\delta \boldsymbol{x}_{i}$ in the state $\boldsymbol{x}\left(t_{i}\right)$ of the dynamic system at a time $t_{i} \in\left[0, t_{\mathrm{f}}\right]$ produce variations $\delta \boldsymbol{u}(t)$ in the optimal control, $\delta \boldsymbol{x}(t)$ in the response, $\delta \boldsymbol{\lambda}(t)$ in the adjoint, $\delta \boldsymbol{\mu}^{L}(t)$ and $\delta \boldsymbol{\mu}^{U}(t)$ in the multipliers of the input bound constraints, and $\delta \overline{\boldsymbol{\nu}}$ in the multipliers of the active terminal constraints $\overline{\boldsymbol{\psi}}$. NE theory provides a means of computing these variations based on the linearization of the first-order NCOs (8) around the extremal path $\boldsymbol{u}_{k}^{*}[23]$.

We assume here that $\boldsymbol{u}_{k}^{*}$ consists of a finite sequence of boundary and interior arcs on $\left[0, t_{\mathrm{f}}\right]$, and that the changes are sufficiently small for the perturbed optimal control to have the same sequence of boundary and interior arcs as well as the same active terminal constraints as with the nominal solution $\boldsymbol{u}_{k}^{*}(t)$. Note that, in general, the switching times between the arcs themselves vary when the initial conditions are modified. Because such switching time variations are difficult to determine and complicate the application of NE control, a common simplification in practice consists in 'freezing' the switching times at their nominal values, updating the control values only between these fixed times [38]. In the context of the two-layer architecture (Fig. 2), this simplification is further justified by the fact that the switching times are adapted by the run-to-run optimizer in the outer layer. An additional key assumption is that the second-order sufficiency conditions (SOSCs) given in [39, 40] for regularity and differentiability of the optimal solution hold.

Under these assumptions, NE control corrections $\delta \boldsymbol{u}(t), t_{i} \leq t \leq t_{\mathrm{f}}$, in response to a state variation $\delta \boldsymbol{x}_{i}$ at any intermediate time $t_{i} \in\left[0, t_{\mathrm{f}}\right]$, can be expressed in the form

$$
\delta \boldsymbol{u}(t)=-\boldsymbol{C}^{*}(t)^{-1}\left[\boldsymbol{A}^{*}(t) \delta \boldsymbol{\lambda}(t)+\boldsymbol{B}^{*}(t) \delta \boldsymbol{x}(t)\right],
$$

where $\delta \boldsymbol{x}(t)$ and $\delta \boldsymbol{\lambda}(t)$ satisfy the following linear TPBVP:

$$
\begin{aligned}
\left(\begin{array}{c}
\delta \dot{\boldsymbol{x}}(t) \\
\delta \dot{\boldsymbol{\lambda}}(t)
\end{array}\right)= & \boldsymbol{\Delta}^{*}(t)\left(\begin{array}{c}
\delta \boldsymbol{x}(t) \\
\delta \boldsymbol{\lambda}(t)
\end{array}\right), \quad \text { a.e. } t \in\left[t_{i}, t_{\mathrm{f}}\right] \\
\text { with: } \quad & \delta \boldsymbol{x}\left(t_{i}\right)=\delta \boldsymbol{x}_{i}, \quad\left[\overline{\boldsymbol{\psi}}_{\boldsymbol{x}}^{*}\right]_{t_{\mathrm{f}}} \delta \boldsymbol{x}\left(t_{\mathrm{f}}\right)=\mathbf{0} \\
& \delta \boldsymbol{\lambda}\left(t_{\mathrm{f}}\right)=\left[\Phi_{\boldsymbol{x} \boldsymbol{x}}^{*}\right]_{t_{\mathrm{f}}} \delta \boldsymbol{x}\left(t_{\mathrm{f}}\right)+\left[\overline{\boldsymbol{\psi}}_{\boldsymbol{x}}^{*}\right]_{t_{\mathrm{f}}}^{\top} \delta \overline{\boldsymbol{\nu}}_{k}^{*} .
\end{aligned}
$$

A derivation for these expressions, including the expression of the matrix $\boldsymbol{\Delta}^{*}(t)$, is reported in Appendix A. 
As part of the SOSCs in $[39,40]$ is the condition that the Hamiltonian function $\mathcal{H}$ is regular. See $[38,41]$ for a possible extension of NE control to singular control problems, both in scalar and vector control problems. See also [42] for NE control of state path-constrained problems. Even though possible, the presence of singular extremals and state path constraints make the formulation of NE controller more involved. These difficulties can be alleviated by applying direct optimal control methods, as described later on in Sect. 4.

Implementation of NE Control The linear TPBVP (10) can be used to calculate the NE control corrections $\delta \boldsymbol{u}(t), t_{i} \leq t \leq t_{\mathrm{f}}$, in either one of two ways:

i. The state variations $\delta \boldsymbol{x}_{i}$ are available at discrete time instants $t_{i} \in\left[0, t_{\mathrm{f}}\right]$, in which case the discrete feedback control can be obtained by directly re-solving the TPBVP. This can be done efficiently via a shooting method as described in [43];

ii. The state variations $\delta \boldsymbol{x}_{i}$ are available in continuous time, in which case the backward sweep method can be used to derive an explicit feedback control law. This approach is thoroughly described in [23].

The focus in this section and in the ensuing case study is on the former approach, which is summarized next. The boundary conditions of the TPBVP (10) can be rewritten in the form:

$$
\left(\begin{array}{ll}
\boldsymbol{I} & \mathbf{0} \\
\mathbf{0} & \mathbf{0} \\
\mathbf{0} & \mathbf{0}
\end{array}\right)\left(\begin{array}{c}
\delta \boldsymbol{x}\left(t_{i}\right) \\
\delta \boldsymbol{\lambda}\left(t_{i}\right)
\end{array}\right)+\left(\begin{array}{cc}
\mathbf{0} & \mathbf{0} \\
-\left[\Phi_{\boldsymbol{x} \boldsymbol{x}}^{*}\right]_{t_{\mathrm{f}}} & \boldsymbol{I} \\
{\left[\overline{\boldsymbol{\psi}}_{\boldsymbol{x}}^{*}\right]_{t_{\mathrm{f}}}} & \mathbf{0}
\end{array}\right)\left(\begin{array}{l}
\delta \boldsymbol{x}\left(t_{\mathrm{f}}\right) \\
\delta \boldsymbol{\lambda}\left(t_{\mathrm{f}}\right)
\end{array}\right)=\left(\begin{array}{c}
\delta \boldsymbol{x}_{i} \\
{\left[\overline{\boldsymbol{\psi}}_{\boldsymbol{x}}^{*}\right]_{t_{\mathrm{f}}}^{\top} \delta \overline{\boldsymbol{\nu}}} \\
\mathbf{0}
\end{array}\right) .
$$

The shooting approach proceeds by guessing the missing initial (or terminal) conditions in (11), and adjusting them in such a way that the corresponding terminal (or initial) conditions are satisfied [see, e.g., 43]. Given the guess $\delta \boldsymbol{\lambda}\left(t_{i}\right)=\delta \boldsymbol{\lambda}_{i}$ for the adjoint variations at time $t_{i}$, the (unique) solution to the (linear) ODE (10) is of the form:

$$
\left(\begin{array}{l}
\delta \boldsymbol{x}(t) \\
\delta \boldsymbol{\lambda}(t)
\end{array}\right)=\underbrace{\left(\begin{array}{cc}
\boldsymbol{\Upsilon}_{1}^{*}\left(t ; t_{i}\right) & \boldsymbol{\Upsilon}_{2}^{*}\left(t ; t_{i}\right) \\
\boldsymbol{\Upsilon}_{3}^{*}\left(t ; t_{i}\right) & \mathbf{\Upsilon}_{4}^{*}\left(t ; t_{i}\right)
\end{array}\right)}_{=: \boldsymbol{\Upsilon}^{*}\left(t ; t_{i}\right)}\left(\begin{array}{c}
\delta \boldsymbol{x}_{i} \\
\delta \boldsymbol{\lambda}_{i}
\end{array}\right)
$$

where the transition matrix $\mathbf{\Upsilon}^{*}\left(t ; t_{i}\right)$ is obtained as the solution to the variational equation

$$
\frac{\partial}{\partial t} \mathbf{\Upsilon}^{*}\left(t ; t_{i}\right)=\boldsymbol{\Delta}^{*}(t) \mathbf{\Upsilon}^{*}\left(t ; t_{i}\right), t_{i} \leq t \leq t_{\mathrm{f}} ; \quad \text { with } \mathbf{\Upsilon}^{*}\left(t_{i} ; t_{i}\right)=\boldsymbol{I}
$$


Substituting (12) into (11) leads to the following linear algebraic system in the variables $\left(\delta \boldsymbol{\lambda}_{i}, \delta \overline{\boldsymbol{\nu}}\right)$ :

$$
\begin{aligned}
& \left(\begin{array}{cc}
{\left[\Phi_{\boldsymbol{x} \boldsymbol{x}}^{*}\right]_{t_{\mathrm{f}}} \mathbf{\Upsilon}_{2}^{*}\left(t_{\mathrm{f}} ; t_{i}\right)-\mathbf{\Upsilon}_{4}^{*}\left(t_{\mathrm{f}} ; t_{i}\right)} & {\left[\overline{\boldsymbol{\psi}}_{\boldsymbol{x}}^{*}\right]_{t_{\mathrm{f}}}^{\boldsymbol{\top}}} \\
{\left[\overline{\boldsymbol{\psi}}_{\boldsymbol{x}}^{*}\right]_{t_{\mathrm{f}}} \mathbf{\Upsilon}_{2}^{*}\left(t_{\mathrm{f}} ; t_{i}\right)} & \mathbf{0}
\end{array}\right)\left(\begin{array}{c}
\delta \boldsymbol{\lambda}_{i} \\
\delta \overline{\boldsymbol{\nu}}
\end{array}\right) \\
& =-\left(\begin{array}{c}
{\left[\Phi_{\boldsymbol{x} \boldsymbol{x}}^{*}\right]_{t_{\mathrm{f}}} \boldsymbol{\Upsilon}_{1}^{*}\left(t_{\mathrm{f}} ; t_{i}\right)-\boldsymbol{\Upsilon}_{3}^{*}\left(t_{\mathrm{f}} ; t_{i}\right)} \\
{\left[\overline{\boldsymbol{\psi}}_{\boldsymbol{x}}^{*}\right]_{t_{\mathrm{f}}} \boldsymbol{\Upsilon}_{1}^{*}\left(t_{\mathrm{f}} ; t_{i}\right)}
\end{array}\right) \delta \boldsymbol{x}_{i} .
\end{aligned}
$$

For given state variations $\delta \boldsymbol{x}_{i}$, the solution to the linear algebraic system (14) thus provides the corresponding adjoint initial variation $\delta \boldsymbol{\lambda}_{i}$ and multiplier variation $\delta \overline{\boldsymbol{\nu}}$. In turn, the NE control variation can be calculated from (9) as

$$
\delta \boldsymbol{u}(t)=-\boldsymbol{C}^{*}(t)^{-1}\left(\boldsymbol{B}^{*}(t) \boldsymbol{A}^{*}(t)\right) \boldsymbol{\Upsilon}^{*}\left(t ; t_{i}\right)\left(\begin{array}{c}
\delta \boldsymbol{x}_{i} \\
\delta \boldsymbol{\lambda}_{i}
\end{array}\right), \quad t \in\left[t_{i}, t_{\mathrm{f}}\right]
$$

Because implementing the full NE correction (15) can lead to inaccurate control action, especially in the presence of large model mismatch, the control corrections can be simply filtered as $\boldsymbol{W}_{\boldsymbol{u}} \delta \boldsymbol{u}(t)$, with $\boldsymbol{W}_{\boldsymbol{u}}$ a gain matrixtypically a diagonal matrix with entries $\omega_{u_{j}}$ such that $0<\omega_{u_{j}} \leq 1$, for each $j=1, \ldots, n_{u}$.

Integration of NE Control with Run-to-Run Constraint Adaptation Following the two-layer architecture given in Fig. 2, a detailed implementation procedure for the indirect two-layer scheme is given in Algorithm 1.

The computational burden of the outer layer in the integrated scheme is clearly similar to that of run-to-run constraint adaptation. On the other hand, the computational effort required to compute the NE updates in the inner layer is mostly limited to solving the linear algebraic system (14) and is thus minimal. This is because the transition matrix $\Upsilon^{*}\left(t ; t_{i}\right)$ needed to construct this linear algebraic system can be computed from the off-line solution of the optimal control problem (1)-(4)\&(6) for each sampling time $t_{i}$; see Step 3 in Algorithm 1.

The indirect two-layer scheme is demonstrated on a case study in the next section. 


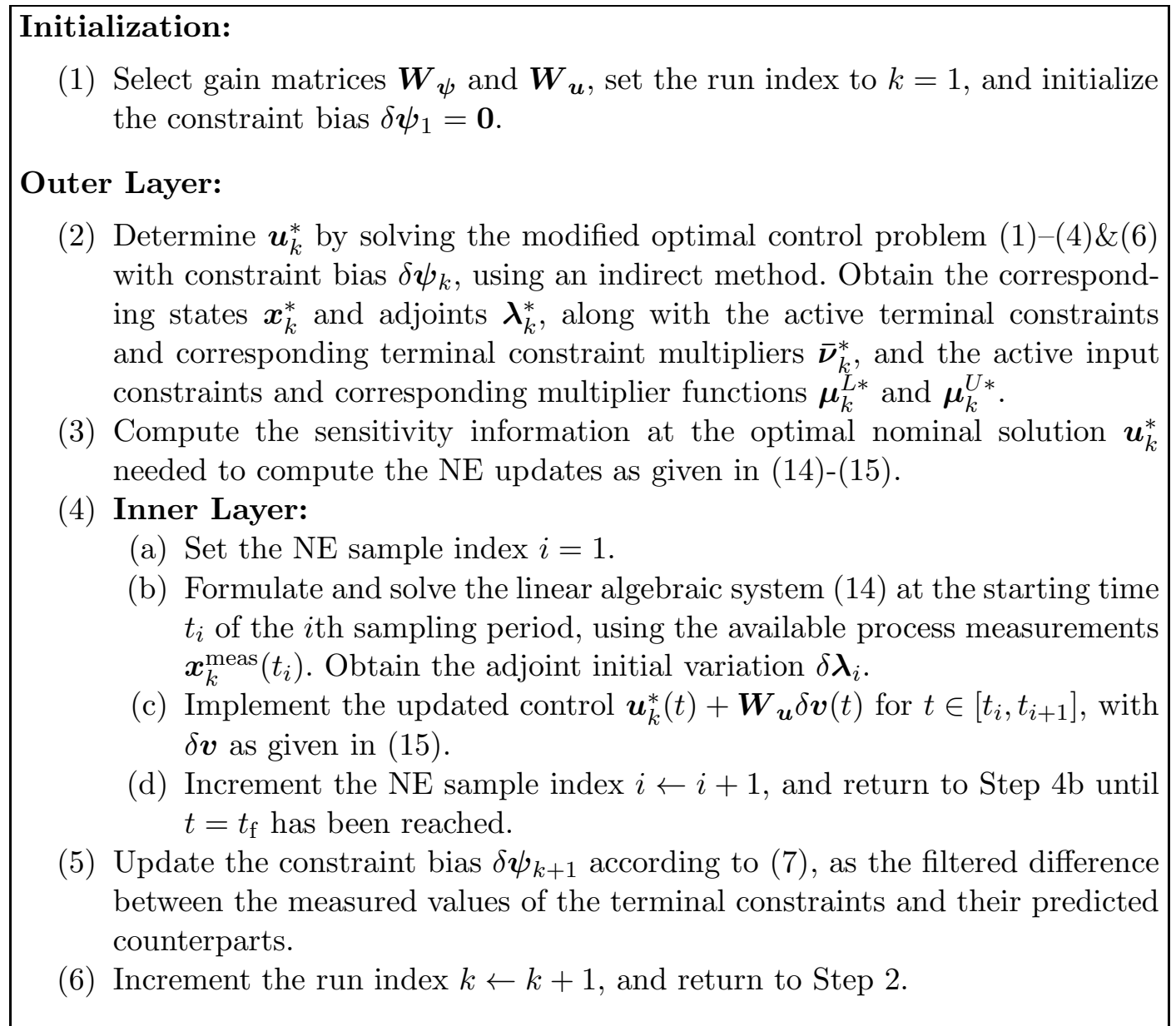

Algorithm 1: Indirect two-layer integrated scheme.

\section{Case Study 1: Connected Tanks with Liquid Interaction}

The level control of a two-connected-tank system with liquid interaction is considered to illustrate the integrated two-layer architecture based on an indirect approach. Comparisons are made against both run-to-run constraint adaptation and NE control alone. These three optimization schemes are first tested in numerical simulations and then validated experimentally.

Given the two-tank system depicted in Fig. 3, the goal is to control the transition to a specified level $h_{2}^{\text {ref }}$ in the tank on the right, by manipulating the inlet flow $u(t)\left[\mathrm{mL} \mathrm{s}^{-1}\right]$ pumped into the tank on the left, over several runs. The outflow from the second tank is regulated by the half-opened valve $k_{22}$ and the two tanks interact through the valve $k_{11}$ at their base. The objective function is so defined as to minimize the integral square error (ISE) between $h_{2}(t)$ and $h_{2}^{\text {ref }}$ :

$$
\min _{u} \int_{0}^{t_{\mathrm{f}}} q\left[h_{2}(t)-h_{2}^{\mathrm{ref}}\right]^{2}+r[u(t)]^{2} \mathrm{~d} t
$$


with weighting constants $r=0.001$ and $q=1000$, and the final time set to $t_{\mathrm{f}}=500 \mathrm{~s}$. Note that the integral term $\int_{0}^{t_{\mathrm{f}}} r[u(t)]^{2} \mathrm{~d} t$ augments the original objective function in order to make the control problem non-singular. This way $\mathcal{H}_{u}$ depends on the control variable and the Hamiltonian $\mathcal{H}$ is regular.

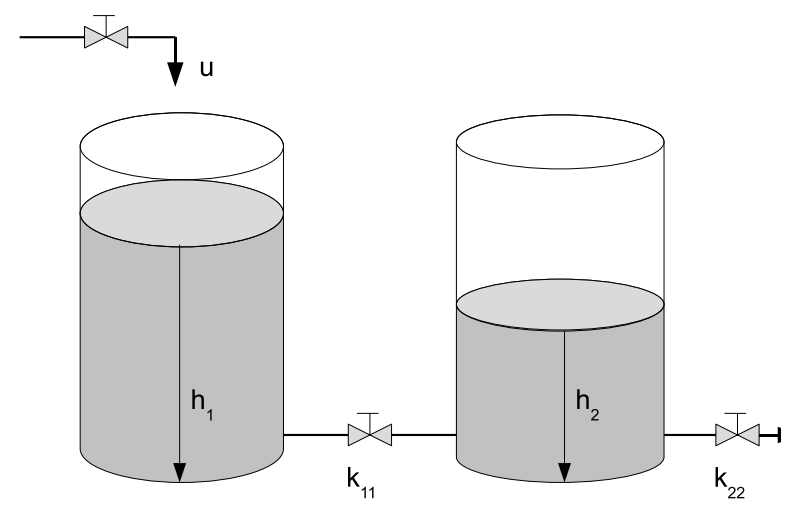

Fig. 3. Configuration of the two tanks connected in series.

The inlet flow $u(t)$ is bounded as:

$$
0 \leq u(t) \leq 100\left[\mathrm{~mL} \mathrm{~s}^{-1}\right]
$$

and a terminal tank-level constraint is imposed as:

$$
h_{2}\left(t_{\mathrm{f}}\right)=h_{2}^{\mathrm{ref}},
$$

with the desired level in the second tank set to $h_{2}^{\text {ref }}=25 \mathrm{~cm}$.

Assuming a constant liquid density and vertical walls in both tanks, a mathematical model is obtained from mass balances and Bernoulli's equation as follows:

$$
\begin{aligned}
& \dot{h}_{1}(t)=\frac{u(t)}{A_{1}}-\frac{k_{11}}{A_{1}} \sqrt{h_{1}(t)-h_{2}(t)} \\
& \dot{h}_{2}(t)=\frac{k_{11}}{A_{2}} \sqrt{h_{1}(t)-h_{2}(t)}-\frac{k_{22}}{A_{2}} \sqrt{h_{2}},
\end{aligned}
$$

where the state variables $h_{1}(t)$ and $h_{2}(t)$ are levels $[\mathrm{cm}]$ in the left tank and in the right tank, respectively; the constants $A_{1}$ and $A_{2}$ defines cross-sectional area of tank bases $\left[\mathrm{cm}^{2}\right] ; k_{11}$ and $k_{22}$ are valve constants $\left[\mathrm{cm}^{2.5} \mathrm{~s}^{-1}\right]$. The initial levels corresponds to a constant inlet flow of $u=25 \mathrm{~mL} \mathrm{~s}^{-1}$, and are given by $h_{1}(0)=16 \mathrm{~cm}$ and $h_{2}(0)=8 \mathrm{~cm}$. The numerical values of the constants are $A_{1}=A_{2}=154 \mathrm{~cm}^{2}, k_{11}=10.68 \mathrm{~cm}^{2.5} \mathrm{~s}^{-1}$, and $k_{22}=7.5 \mathrm{~cm}^{2.5} \mathrm{~s}^{-1}$.

The optimal control trajectory for this problem is shown in Fig. 4. This solution was determined by first applying a direct sequential approach with a 
piecewise-constant control parameterization [28, 44], and then refining the solution using an indirect shooting approach [23]. It consists of an upper bound, a lower bound, and an interior arc. The first two bangs allow reaching the specified reference in minimum time, after which the flow rate is maintained constant to keep the water level at this level.

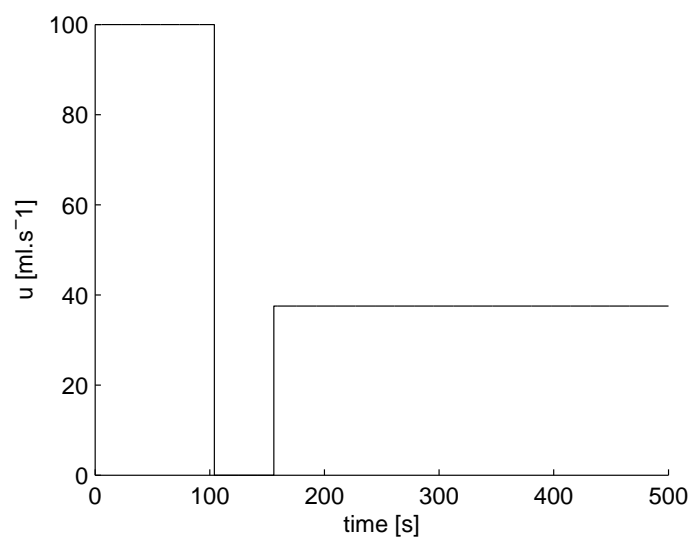

Fig. 4. Nominal optimal control trajectory for the two-tank system.

\subsection{Performance of Indirect Two-Layer Scheme}

In order to simulate the real behavior of the process, the valve constants are perturbed to the values $k_{11}=10.08 \mathrm{~cm}^{2.5} \mathrm{~s}^{-1}$ and $k_{22}=8.82 \mathrm{~cm}^{2.5} \mathrm{~s}^{-1}$, which indirectly perturbs the initial conditions $h_{1}(0)$ and $h_{2}(0)$ as well. When the open-loop control profile is applied, such a mismatch between the nominal and perturbed models leads to a violation of the terminal constraint and an increase in the ISE objective function.

The measured outputs are the two states $h_{1}(t)$ and $h_{2}(t)$, with added Gaussian white noise of relative variance $1 \%$, and the measurement period is $1 \mathrm{~s}$. Run-torun constraint adaptation is initialized with a constraint bias of $\delta \psi_{1}=0$, and filter gains of $W_{\psi}=0.6$ and $W_{\psi}=0.4$ are used in the constraint-adaptation scheme and in the two-layer scheme, respectively. On the other hand, filter gains of $W_{u}=1$ are used for the NE updates.

A comparison of the performance of all three optimization schemes during the first 15 runs is shown on the left plot of Fig. 5. Observe first that the constraintadaptation scheme starts relatively far from the desired reference level $h_{2}^{\text {ref }}$ in the first run, compared to the NE controller or the two-layer scheme. It takes about 5 runs for constraint adaptation alone to reach a neighborhood of the terminal level constraint. The integrated two-layer scheme starts relatively closer to the terminal constraint and converges to the desired vale within about 5 runs too, despite a smaller filter gain $W_{\psi}=0.4$. Moreover, due to the on-line corrective action in the inner layer, the effect of the measurement noise on the final level $h_{2}\left(t_{\mathrm{f}}\right)$ is much smaller with that scheme. Notice also 

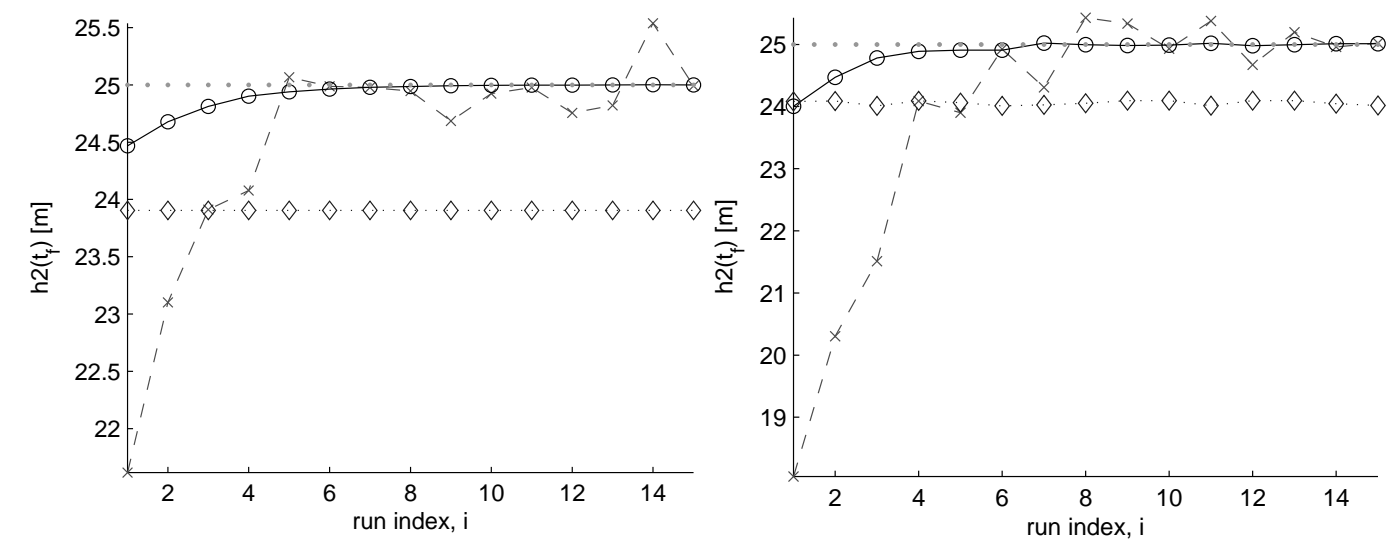

Fig. 5. Compared evolution of the terminal level $h_{2}\left(t_{\mathrm{f}}\right)$ for the three optimization schemes. Left plot: Simulated reality; Right plot: Experimental results. Dashed line with crosses: run-to-run constraint adaptation alone; Dotted line with diamonds: NE controller alone; Solid line with circles: integrated two-layer optimization scheme; Thick dotted line: desired level $h_{2}^{\text {ref }}$.
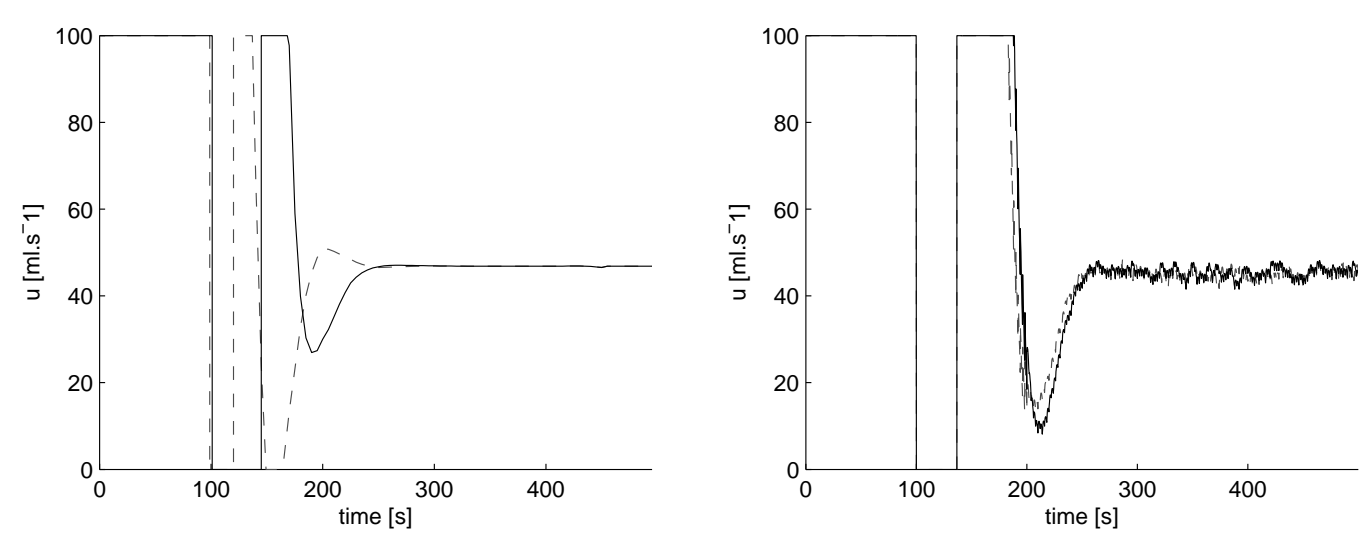

Fig. 6. Adapted control profiles with the integrated two-layer scheme during runs 1 and 15. Left plot: Simulated reality; Right plot: Experimental results; Dashed line: 1 st run; Solid line: 15 th run.

that the variation of the constraint bias $\delta \psi$ is significantly smaller with the two-layer scheme than with constraint adaptation alone.

The control profiles obtained with the integrated two-layer optimization scheme after 1 and 15 runs are shown on left plot of Fig. 6. Both profiles are comprised of a number of bangs prior to the interior arc, similar to the nominal solution in Fig. 4. Note, in particular, that the control along the unconstrained arc is no longer constant due to the corrective actions of the NE controller. Moreover, the switching times too are modified as a result of the corrective action of the run-to-run constraint adaptation scheme in the outer layer. 


\subsection{Experimental Validation}

The experiments are carried out on an Amira DTS200 device, which is shown in Fig. 7. The device consists of 3 connected tube-shaped tanks connected through their bases and comprises six valves to regulate the outflows. The levels are measured by pressure sensors located at the bottom of each tank, with a sampling time of $1 \mathrm{~s}$. Also, two inlet flows are available, with pumps feeding the liquid to the first and third tanks. Only the first two tanks are used in this case study, as depicted in Fig. 3.
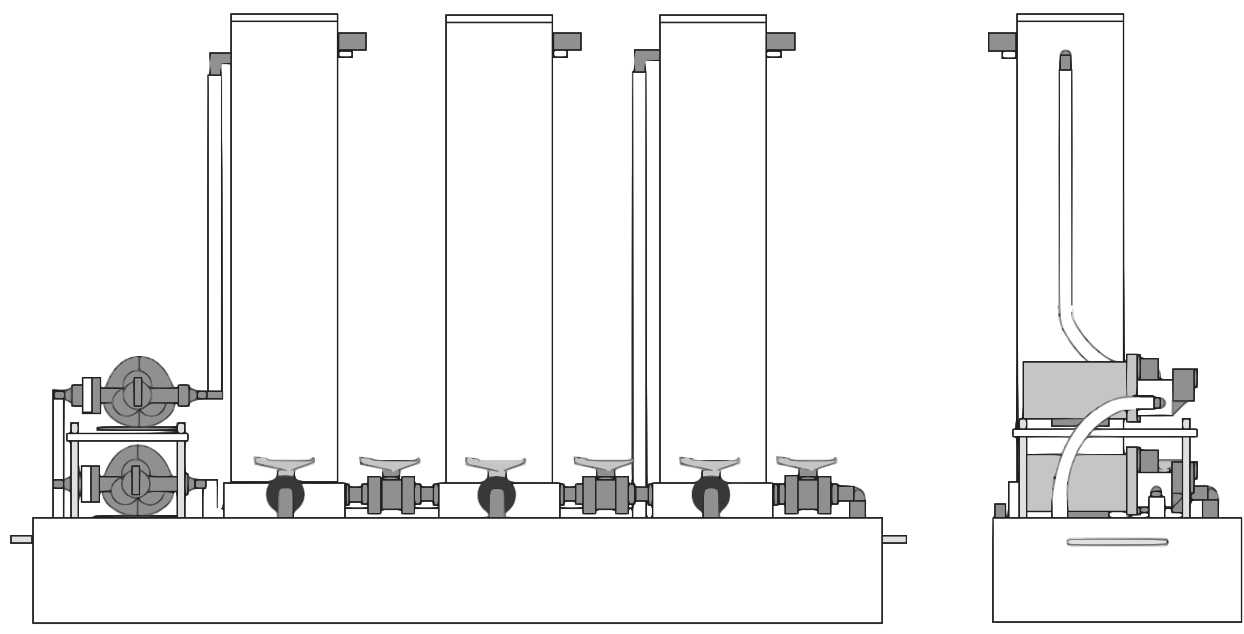

Fig. 7. Amira DTS200 - Process for level control of tanks.

The nominal solution is first obtained for certain positions of the outflow valves (leakages). The outflow is then increased, which causes small variations in the initial conditions too. The conversion between measured outputs (in volts) and levels (in centimeters) is another source of uncertainty in the experimental system. Such mismatch between the nominal model and the experimental system leads to a violation of the terminal constraint and an increase in the ISE objective function.

A comparison of the three optimization schemes during the first 15 runs in terms of their performance is shown on the right plot of Fig. 5. A similar behavior as in numerical simulations is observed. In the first run, the NE controller and the two-layer scheme allow a closer start from the desired reference level $h_{2}^{\text {ref }}$ compared to constraint adaptation alone. The NE controller is able to recover some of the constraint violation, but not all of it. During the following 5-6 runs, the two-layer scheme slowly increases the terminal constraint until the desired level is reached. This is about the same number of runs taken by the run-to-run constraint adaptation scheme to reach the constraint, yet with a higher value of the filter gain and a larger bias correction. Moreover, the two-layer scheme exhibits a lower sensitivity to measurement noise than run-to-run adaptation, due to the corrective effect of the NE controller in 
the inner layer. The control profiles obtained with the integrated two-layer optimization scheme after 1 and 15 runs are shown on the right plot of Fig. 6 .

\section{Direct Two-Layer Scheme}

Direct optimal control methods approximate the optimal control problem by a finite-dimensional nonlinear programming (NLP) problem, which can then be solved using standard numerical optimization algorithms. This approximation relies on a parameterization of the control trajectories, and therefore direct methods are less rigorous than indirect methods in this regard. In practice, however, the resulting performance loss is hardly noticeable when a tailored parameterization is used or by applying an adaptive parameterization approach [45]. Besides, singular control problems as well as path-constrained problems can be handled more conveniently by direct methods.

Three main variants of this method are direct single shooting, direct multiple shooting, and orthogonal collocation [see, e.g., 27, 28]. The focus in this section is on the former approach, also known as direct sequential approach, but the following ideas can be readily extended to the other two approaches. Some background elements on direct single shooting and NE updates are first reviewed, before stating the direct two-layer optimization algorithm.

Direct Single Shooting Given a finite number $M \geq 1$ of piecewisecontinuous basis functions $\chi^{i}:\left[0, t_{\mathrm{f}}\right] \rightarrow \mathbb{R}^{n_{u}}, i=1, \ldots, M$, the control trajectories are approximated as

$$
\boldsymbol{u}(t)=\mathcal{U}(t, \boldsymbol{v}):=\sum_{i=1}^{M} \boldsymbol{v}^{i} \chi^{i}(t), \quad t \in\left[0, t_{\mathrm{f}}\right]
$$

Usual control parameterizations include piecewise constant and piecewise linear functions. The coefficients $\boldsymbol{v}=\left[\boldsymbol{v}^{1^{\top}} \cdots \boldsymbol{v}^{M^{\top}}\right]^{\top} \in \mathbb{R}^{n_{v}}$, with $n_{v}=n_{u} \cdot M$, become the new decision variables in a finite-dimensional optimization problem, whose solution approximates that of the original optimal control problem:

$$
\begin{array}{cl}
\min _{\boldsymbol{v}} & \phi\left(\boldsymbol{x}\left(t_{\mathrm{f}}\right)\right)+\int_{0}^{t_{\mathrm{f}}} L(\boldsymbol{x}(t), \mathcal{U}(t, \boldsymbol{v})) \mathrm{d} t \\
\text { s.t. } & \dot{\boldsymbol{x}}(t)=\boldsymbol{F}(\boldsymbol{x}(t), \mathcal{U}(t, \boldsymbol{v})), \quad 0 \leq t \leq t_{\mathrm{f}} \\
& \boldsymbol{x}(0)=\boldsymbol{x}_{0} \\
& \boldsymbol{u}^{L} \leq \mathcal{U}(t, \boldsymbol{v}) \leq \boldsymbol{u}^{U} \\
& \boldsymbol{\psi}\left(\boldsymbol{x}\left(t_{\mathrm{f}}\right)\right) \leq \delta \boldsymbol{\psi}_{k} .
\end{array}
$$


In particular, the response of the dynamic system is regarded as a function of the control parameterization coefficients, and the evaluation of the objective and constraint functions in the discretized NLP is via the numerical integration of the differential equations. Moreover, in using derivative-based NLP techniques such as SQP, the first-order derivatives - and the second-order derivatives too if necessary - can be obtained from the numerical integration of the associated state-sensitivity or adjoint equations [28].

Neighboring-Extremal Updates In the analysis that follows, we regard the optimization problem $(22)-(26)$ on the reduced time horizon $\left[t_{i}, t_{\mathrm{f}}\right]$ as a parametric NLP problem in the initial state conditions $\boldsymbol{x}\left(t_{i}\right)=\boldsymbol{x}_{i}$ of the form:

$$
\begin{array}{ll}
\min _{\boldsymbol{v}} & f\left(\boldsymbol{v}, \boldsymbol{x}_{i}\right) \\
\text { s.t. } & \boldsymbol{g}\left(\boldsymbol{v}, \boldsymbol{x}_{i}\right) \leq \mathbf{0} .
\end{array}
$$

Let $\boldsymbol{v}_{k}^{*}$ be an optimal solution of the problem (22)-(26) on the full horizon $\left[0, t_{\mathrm{f}}\right]$, and let $\boldsymbol{x}_{k}^{*}$ be the corresponding optimal response of the dynamic system. By Bellman's principle, $\boldsymbol{v}_{k}^{*}$ must also be an optimal solution of the parametric NLP problem (27) on $\left[t_{i}, t_{\mathrm{f}}\right]$ for the initial conditions $\boldsymbol{x}\left(t_{i}\right)=\boldsymbol{x}_{k}^{*}\left(t_{i}\right)$.

Provided that the gradients of the constraints in (27) are linearly independent at $\boldsymbol{v}_{k}^{*}$ (linear independence constraint qualification, LICQ), there exist (unique) KKT multipliers $\gamma_{k}^{*}$ such that the following first-order NCOs hold:

$$
\begin{aligned}
& \mathcal{L}_{\boldsymbol{v}}\left(\boldsymbol{v}_{k}^{*}, \boldsymbol{\gamma}_{k}^{*}, \boldsymbol{x}_{i}\right)=\mathbf{0} \\
& \boldsymbol{g}\left(\boldsymbol{v}_{k}^{*}, \boldsymbol{x}_{i}\right) \leq \mathbf{0}, \quad \boldsymbol{\gamma}_{k}^{* \top} \boldsymbol{g}\left(\boldsymbol{v}_{k}^{*}, \boldsymbol{x}_{i}\right)=\mathbf{0}, \quad \gamma_{k}^{*} \geq \mathbf{0},
\end{aligned}
$$

with the Lagrangian function defined as $\mathcal{L}\left(\boldsymbol{v}, \boldsymbol{\gamma}, \boldsymbol{x}_{i}\right):=f\left(\boldsymbol{v}, \boldsymbol{x}_{i}\right)+\boldsymbol{\gamma}^{\top} \boldsymbol{g}\left(\boldsymbol{v}, \boldsymbol{x}_{i}\right)$.

Small changes $\delta \boldsymbol{x}_{i}$ in the state of the dynamic system at a time $t_{i} \in\left[0, t_{\mathrm{f}}\right]$ produce variations $\delta \boldsymbol{v}$ in the optimal control parameterization coefficients, and $\delta \boldsymbol{\gamma}$ in the constraint multipliers. Under the extra assumptions that strong second-order sufficient conditions for optimality and strict complementarity slackness are satisfied at the nominal solution $\boldsymbol{v}_{k}^{*}$, the variations $\delta \boldsymbol{v}$ and $\delta \bar{\gamma}-$ corresponding to the active constraints $\overline{\boldsymbol{g}}\left(\boldsymbol{v}_{k}^{*}, \boldsymbol{x}_{i}\right)=\mathbf{0}$ - can be estimated via the linearization of the NCOs (29) around $\boldsymbol{v}_{k}^{*}$ and the solution of a simple linear algebraic system as [24]:

$$
\left(\begin{array}{cc}
\mathcal{L}_{\boldsymbol{v} \boldsymbol{v}}^{*} & \overline{\boldsymbol{g}}_{\boldsymbol{v}}^{* \top} \\
\overline{\boldsymbol{g}}_{\boldsymbol{v}}^{*} & \mathbf{0}
\end{array}\right)\left(\begin{array}{c}
\delta \boldsymbol{v} \\
\delta \bar{\gamma}
\end{array}\right)=-\left(\begin{array}{c}
\mathcal{L}_{\boldsymbol{v} \boldsymbol{x}_{i}}^{*} \\
\overline{\boldsymbol{g}}_{\boldsymbol{x}_{i}}^{*}
\end{array}\right) \delta \boldsymbol{x}_{i}
$$

Tools for computing such NE updates automatically are also becoming available, such as sIPOPT [46]. A limitation of this approach, however, is that an 
actual first-order approximation of the perturbed optimal solution can only be obtained when the set of active constraints remains unchanged. Note that this limitation is shared with the indirect approach outlined earlier in Sect. 4 too.

An improved approach that predicts changes in active constraints, in addition to estimating the variations $\delta \boldsymbol{v}$ and $\delta \boldsymbol{\gamma}$, involves solving the following quadratic programming (QP) problem [47, 48]:

$$
\begin{array}{ll}
\min _{\delta \boldsymbol{v}} & \frac{1}{2} \delta \boldsymbol{v}^{\top} \mathcal{L}_{\boldsymbol{v} \boldsymbol{v}}^{*} \delta \boldsymbol{v}+\delta \boldsymbol{x}_{i}^{\top} \mathcal{L}_{\boldsymbol{x}_{i} \boldsymbol{v}}^{*} \delta \boldsymbol{v}+f_{\boldsymbol{v}}^{* \top} \delta \boldsymbol{v} \\
\text { s.t. } & \boldsymbol{g}^{*}+\boldsymbol{g}_{\boldsymbol{v}}^{*} \delta \boldsymbol{v}+\boldsymbol{g}_{\boldsymbol{x}_{i}}^{*} \delta \boldsymbol{x}_{i} \leq \mathbf{0}
\end{array}
$$

The solution $\delta \boldsymbol{v}$ of this QP problem amounts to taking a Newton step for the equalities part in the first-order NCOs (29) at the nominal solution $\left(\boldsymbol{v}^{*}, \boldsymbol{\gamma}^{*}\right)$, with the added restriction that the constraint are satisfied to first-order and $\hat{\gamma}:=\boldsymbol{\gamma}^{*}+\delta \boldsymbol{\gamma}$ remains nonnegative; that is, the KKT conditions are correct to first-order for this step. In particular, it is not hard to check that a solution pair $(\delta \boldsymbol{v}, \hat{\gamma})$ of $(30)$ corresponds to a solution $(\delta \boldsymbol{v}, \delta \boldsymbol{\gamma})$ of the linear algebraic system (29) if the active set does not change. Notice also the strong similarity with MPC in regards of the formulation and solution of QP problems at each sampling time.

Implementing the full NE correction $\boldsymbol{v}_{k}^{*}+\delta \boldsymbol{v}$ at each sampling time $t_{i}$ yields an accurate approximation of the perturbed optimal solution when the deviations $\delta \boldsymbol{x}_{i}$ are small. For larger changes, however, the NE updates can become inaccurate, e.g. due to the nonlinearities or model mismatch. One way to mitigate these inaccuracies simply consists in filtering the QP solution as $\boldsymbol{v}_{k}^{*}+\boldsymbol{W}_{\boldsymbol{u}} \delta \boldsymbol{v}$, with $\boldsymbol{W}_{\boldsymbol{u}} \in \mathbb{R}^{n_{u} \times n_{u}}$ a (diagonal) gain matrix. A more involved approach proposed by [9] considers multiple QP iterations, similar in essence to the iterations in an SQP algorithm. While these additional iterations reduce the effect of the linearization inherent to NE updates, an additional on-line computational burden here is the need to recompute the first- and directional second-order sensitivity at every iteration.

\section{Integration of NE Updates with Run-to-Run Constraint Adaptation}

A detailed implementation procedure for the direct two-layer scheme is given in Algorithm 2. For simplicity, the NE-QP updates are synchronized with the beginning of every sampling period, and the time delays associated with the measurements and the QP solution are not accounted for. We refer the reader to $[6,9]$ for a discussion of the effect of time delays.

As expected, the direct scheme in Algorithm 2 shares many common steps with the indirect scheme in Algorithm 1. Perhaps the main difference besides the application of a control parameterization is the fact that a QP problem is 


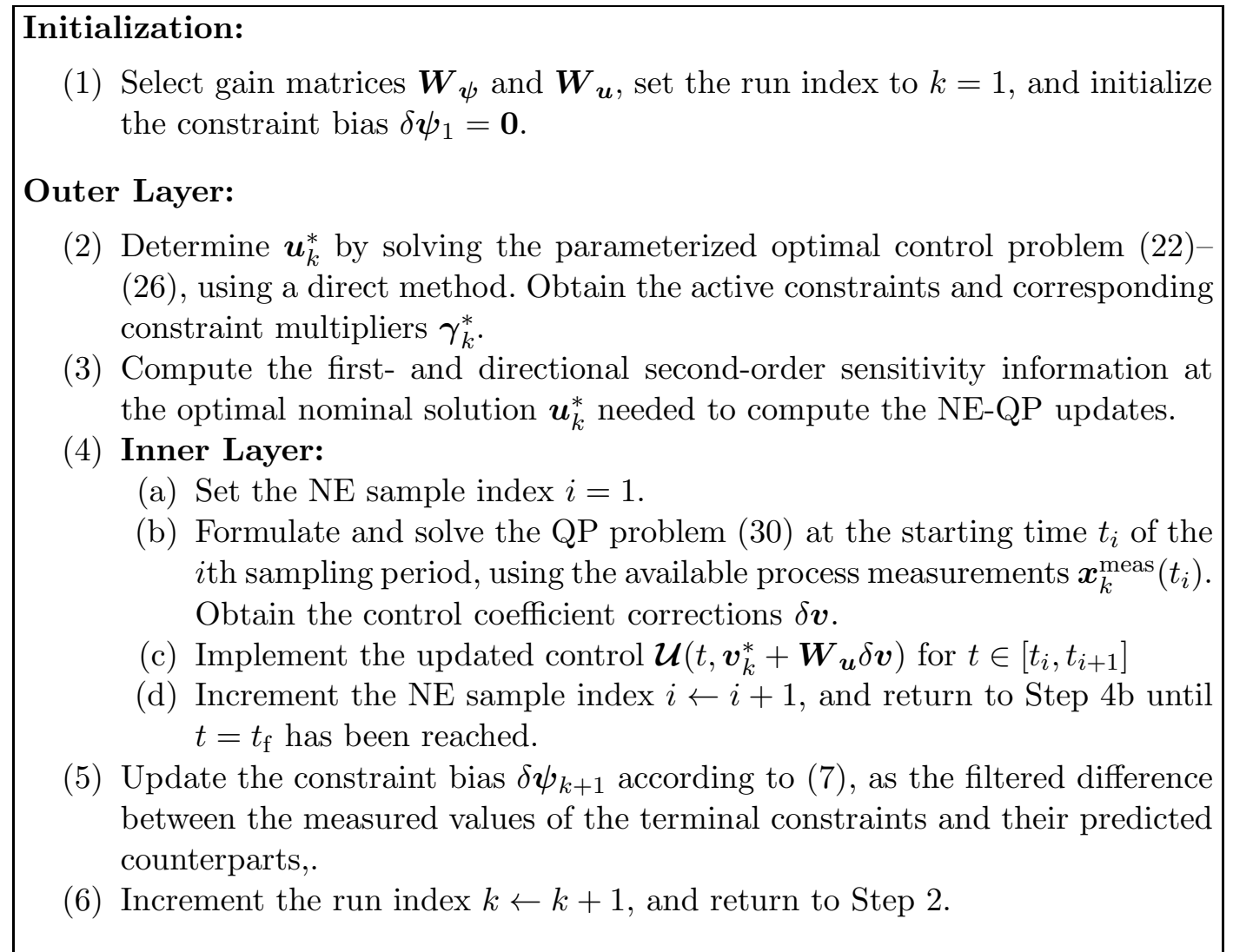

Algorithm 2: Direct two-layer integrated scheme.

now solved in Step 4b of Algorithm 2 as opposed to a simple linear algebraic system in Algorithm 1. A clear benefit of this extra complexity is the ability to track changing active sets. In terms of computational time, the numerical solution of the QP problems (30) at each sampling time $t_{i}$ in Step $4 \mathrm{~b}$ is the dominant computational cost. Nonetheless, efficient methods and tools exist for solving large-scale QP problems, and the QP solvers can also be warmstarted by using the solutions computed at previous sampling times. We also note that the first- and directional second-order sensitivity information around the nominal solution $\boldsymbol{v}_{k}^{*}$ that is needed to construct the QP problems can be computed off-line. Efficient techniques based on forward and reverse sensitivity analysis are available to obtain such information $[49,50]$.

The direct two-layer scheme is illustrated on a case study in the following section.

\section{Case Study 2: Acetoacetylation of Pyrrole in Fed-batch Reactor}

A semi-batch reactor example inspired from $[32,51]$ is considered to illustrate the integrated two-layer scheme using the direct approach. The reaction sys- 
tem is the acetoacetylation of pyrrole with diketene and consists of 4 reactions:

$$
\begin{array}{r}
\mathrm{A}+\mathrm{B} \stackrel{k_{1}}{\longrightarrow} \mathrm{C} \\
2 \mathrm{~B} \stackrel{k_{2}}{\longrightarrow} \mathrm{D} \\
\mathrm{B} \stackrel{k_{3}}{\longrightarrow} \mathrm{E} \\
\mathrm{C}+\mathrm{B} \stackrel{k_{4}}{\longrightarrow} \mathrm{F},
\end{array}
$$

with A: pyrrole; B: diketene; C: 2-acetoacetyl pyrrole; D: dehydroacetic acid; E: oligomers; F: undesired by-product. A first-principles model for the semi-batch reactor is as follows:

$$
\begin{aligned}
\dot{c}_{\mathrm{A}} & =-k_{1} c_{\mathrm{A}} c_{\mathrm{B}}-\frac{F}{V} c_{\mathrm{A}} \\
\dot{c}_{\mathrm{B}} & =-k_{1} c_{\mathrm{A}} c_{\mathrm{B}}-2 k_{2} c_{\mathrm{B}}^{2}-k_{3} c_{\mathrm{B}}-k_{4} c_{\mathrm{B}} c_{\mathrm{C}}+\frac{F}{V}\left(c_{\mathrm{B}}^{\text {in }}-c_{\mathrm{B}}\right) \\
\dot{c}_{\mathrm{C}} & =k_{1} c_{\mathrm{A}} c_{\mathrm{B}}-k_{4} c_{\mathrm{B}} c_{\mathrm{C}}-\frac{F}{V} c_{\mathrm{C}} \\
\dot{c}_{\mathrm{D}} & =k_{2} c_{\mathrm{B}}^{2}-\frac{F}{V} c_{\mathrm{D}} \\
\dot{V} & =F,
\end{aligned}
$$

where $c_{\mathrm{A}}, c_{\mathrm{B}}, c_{\mathrm{C}}$ and $c_{\mathrm{D}}$ stand for the concentrations of species $\mathrm{A}, \mathrm{B}, \mathrm{C}$ and $\mathrm{D}$, respectively; $V$, the reactor volume; $F$, the inlet flow rate of species $\mathrm{B}$; and $c_{\mathrm{B}}^{\mathrm{in}}$, the concentration of $\mathrm{B}$ in the feed.

Throughout this case study, the full reaction mechanism is considered in the plant simulator, with the corresponding kinetic parameter and initial concentration values given in Table 1 . On the other hand, only the first two reactions $(\mathrm{A}+\mathrm{B} \rightarrow \mathrm{C}$ and $2 \mathrm{~B} \rightarrow \mathrm{D})$ are taken into account in the plant model, while the remaining two reactions are considered to be unknown side reactions $\left(k_{3}=k_{4}=0\right)$. Moreover, the kinetic parameter and initial concentration values in the plant model differ from those in the plant simulator, as given in Table 2. This way, the model has both structural and parametric mismatch.

Table 1

Parameters values and initial conditions in simulated plant.

\begin{tabular}{lrllll}
\hline Parameter & \multicolumn{2}{c}{ Value } & Concentration & \multicolumn{2}{c}{ Value } \\
\hline$k_{1}$ & 0.053 & $\mathrm{~L} \mathrm{~mol}^{-1} \mathrm{~min}^{-1}$ & $c_{\mathrm{A}}(0)$ & 0.72 & $\mathrm{~mol} \mathrm{~L}^{-1}$ \\
$k_{2}$ & 0.128 & $\mathrm{~L} \mathrm{~mol}^{-1} \mathrm{~min}^{-1}$ & $c_{\mathrm{B}}(0)$ & 0.05 & $\mathrm{~mol} \mathrm{~L}^{-1}$ \\
$k_{3}$ & 0.028 & $\mathrm{~min}^{-1}$ & $c_{\mathrm{C}}(0)$ & 0.08 & $\mathrm{~mol} \mathrm{~L}^{-1}$ \\
$k_{4}$ & 0.001 & $\mathrm{~L} \mathrm{~mol}^{-1} \mathrm{~min}^{-1}$ & $c_{\mathrm{D}}(0)$ & 0.01 & $\mathrm{~mol} \mathrm{~L}^{-1}$ \\
$c_{\mathrm{B}}^{\text {in }}$ & 5 & $\mathrm{~mol} \mathrm{~L}^{-1}$ & $V(0)$ & 1 & $\mathrm{~L}$ \\
\hline
\end{tabular}


Table 2

Parameters values and initial conditions in plant model.

\begin{tabular}{lrllll}
\hline Parameter & \multicolumn{2}{c}{ Value } & Concentration & \multicolumn{2}{c}{ Value } \\
\hline$k_{1}$ & 0.06 & $\mathrm{~L} \mathrm{~mol}^{-1} \mathrm{~min}^{-1}$ & $c_{\mathrm{A}}(0)$ & 0.75 & $\mathrm{~mol} \mathrm{~L}^{-1}$ \\
$k_{2}$ & 0.11 & $\mathrm{~L} \mathrm{~mol}^{-1} \mathrm{~min}^{-1}$ & $c_{\mathrm{B}}(0)$ & 0.10 & $\mathrm{~mol} \mathrm{~L}^{-1}$ \\
$k_{3}$ & - & $\mathrm{min}^{-1}$ & $c_{\mathrm{C}}(0)$ & - & $\mathrm{mol} \mathrm{L}^{-1}$ \\
$k_{4}$ & - & $\mathrm{L} \mathrm{mol}^{-1} \mathrm{~min}^{-1}$ & $c_{\mathrm{D}}(0)$ & - & $\mathrm{mol} \mathrm{L}^{-1}$ \\
$c_{\mathrm{B}}^{\text {in }}$ & 5 & $\mathrm{~mol} \mathrm{~L}^{-1}$ & $V(0)$ & 1 & $\mathrm{~L}$ \\
\hline
\end{tabular}

The goal is to determine the feed profile of species B that maximizes the number of moles of $\mathrm{C}$ at final time, while keeping the concentrations of $\mathrm{B}$ and $\mathrm{D}$ at terminal time below specified threshold values:

$$
\begin{aligned}
\max _{F(t)} & c_{\mathrm{C}}\left(t_{\mathrm{f}}\right) V\left(t_{\mathrm{f}}\right) \\
\text { s.t. } & \text { Model }(31-35) \\
& c_{\mathrm{B}}\left(t_{\mathrm{f}}\right) \leq c_{\mathrm{B}}^{\max } \\
& c_{\mathrm{D}}\left(t_{\mathrm{f}}\right) \leq c_{\mathrm{D}}^{\max } \\
& 0 \leq F(t) \leq F^{\max },
\end{aligned}
$$

with the final time $t_{\mathrm{f}}=250 \mathrm{~min}$; the maximal inlet flow rate $F^{\max }=2 \times$ $10^{-3} \mathrm{~L} \mathrm{~min}{ }^{-1}$; and the maximal concentrations of species $\mathrm{B}$ and $\mathrm{D}$ at final time $c_{\mathrm{B}}^{\max }=0.025 \mathrm{~mol} \mathrm{~L}{ }^{-1}$ and $c_{\mathrm{D}}^{\max }=0.15 \mathrm{~mol} \mathrm{~L}{ }^{-1}$.

The optimal control and state trajectories, as obtained with a piecewiseconstant parameterization over 50 equal stages and direct single shooting $[44,52]$, are shown in Figure 8. The optimal performance is $\Phi^{*} \approx$ $0.5081 \mathrm{~mol}$, and the optimal feed profile is comprised of 3 arcs: (i) a boundary arc with $F=F^{\max }$; (ii) an interior (singular) arc, where the feed level is intermediate between 0 and $F^{\max }$; and (iii) another boundary arc with $F=0$. Moreover, both terminal constraints are active in this optimal solution. Also shown in Figure 8 is the solution of the discretized optimal control problem computed with the plant model (i.e., with $k_{3}=k_{4}=0$ ). Open-loop application of the model-based optimum on the simulated plant yields a feasible operation, yet causes a large performance loss with $\Phi \approx 0.4003 \mathrm{~mol}$.

\subsection{Performance of Integrated Two-Layer Scheme}

Since the optimal control problem (36) is singular, a direct, integrated twolayer scheme is implemented. A control parameterization over 50 identical, piecewise-constant stages is used, and within-run corrections based on 

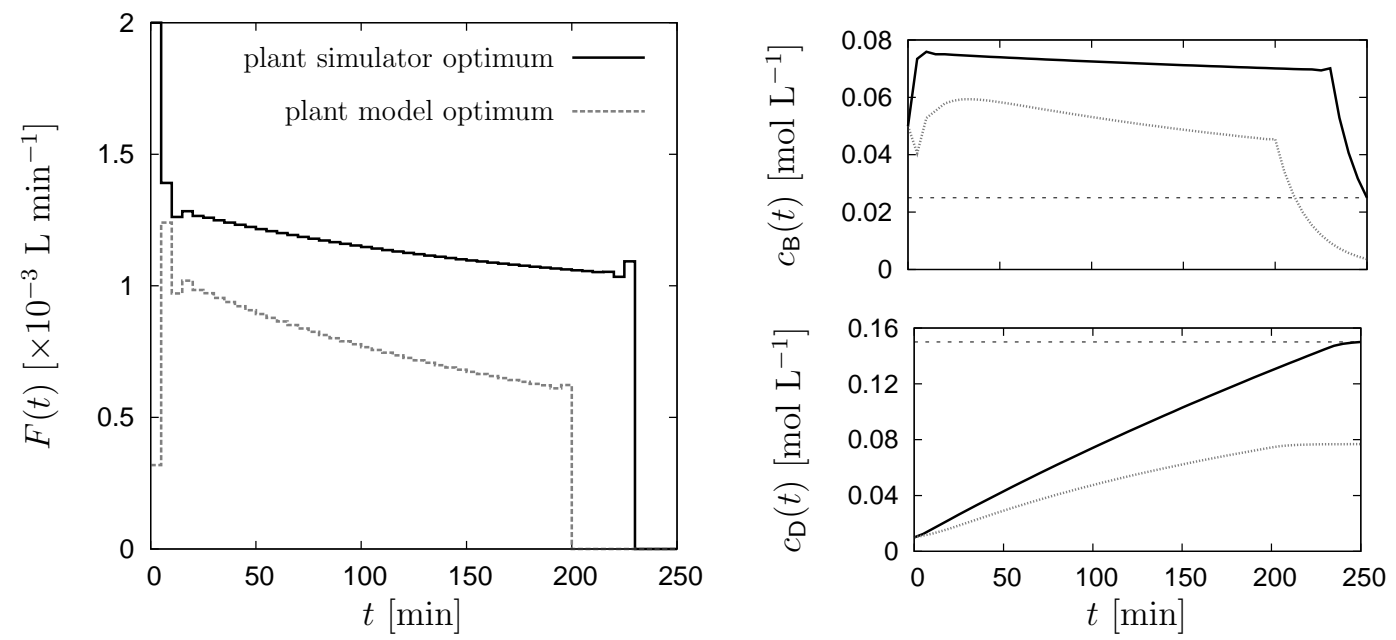

Fig. 8. Discretized optimal control strategy in Problem (36) using piecewise-constant parameterization over 50 stages.

sensitivity-based updates run every 5 minutes. Full-state measurements and no delays are assumed here for simplicity. Run-to-run adaptation is initialized with a constraint bias of $\delta \psi_{1}=0$, and the constraint filter is taken as $W_{\psi}=0.7$ throughout - a value so chosen to reach the terminal-constraint limit as fast as possible, while avoiding oscillations around this limit.

The performance of the integrated scheme is first assessed under noise-free conditions. The results of the first 20 batches are depicted in Figure 9, with the cost function and terminal constraints given in the left and right plots, respectively. Various input-filter parameters $W_{u}=1,0.4,0.2$, and 0 are compared on these plots. Constraint adaptation alone, which corresponds to the input-filter value $W_{u}=0$, is seen to remain feasible in all batches, yet it takes about 8-10 batches to achieve convergence. The use of within-run updates has a large impact in terms of convergence speed, as it reduces the need for large corrections of the constraint biases. Particularly important here is the input-filter parameter $W_{u}$, where a larger value generally promotes faster convergence, but the QP approximation can result in over-corrections when too large a value is used, e.g. with $W_{u}=1.0$. A good trade-off is found to be $W_{u}=0.4$ in this instance.

Accordingly, the cost value of the converged adaptation scheme is found to deteriorate if the input-filter parameter $W_{u}$ is too large, the best performance being obtained with $W_{u}=0.2$ here. This trend is confirmed by inspection of the corresponding (converged) control profiles in Figure 10, where the discrepancy between these profiles and the actual optimal strategy (dotted line) increases with the input-filter parameter $W_{u}$. It is noteworthy that such a behavior is by no means general and the use of fast-sensitivity updates can as well contribute to larger reduction of the optimality gap in other case studies. This behavior can be attributed to the quality of the QP approximations, 

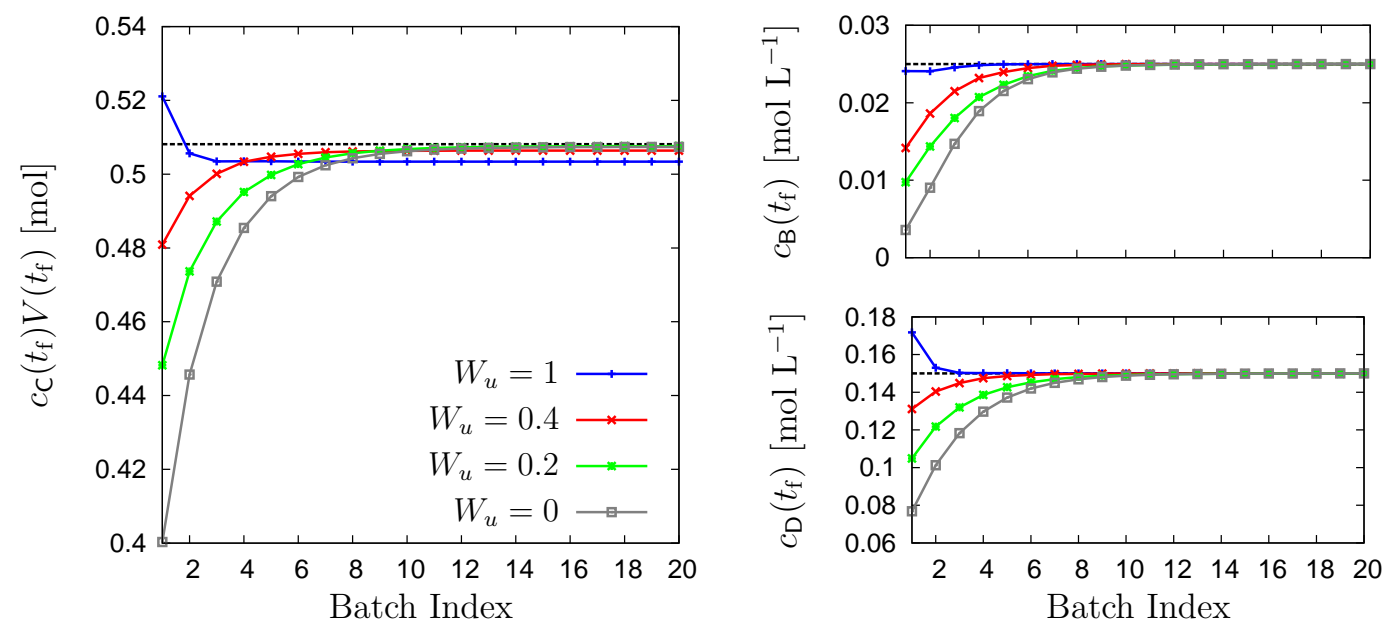

Fig. 9. Compared performance of integrated two-layer schemes with constraint-filter parameter $W_{\psi}=0.7$ and various input-filter parameters $W_{u}=1.0,0.4,0.2$, and 0 .

which typically deteriorate as the model mismatch gets bigger, especially for strongly nonlinear systems.

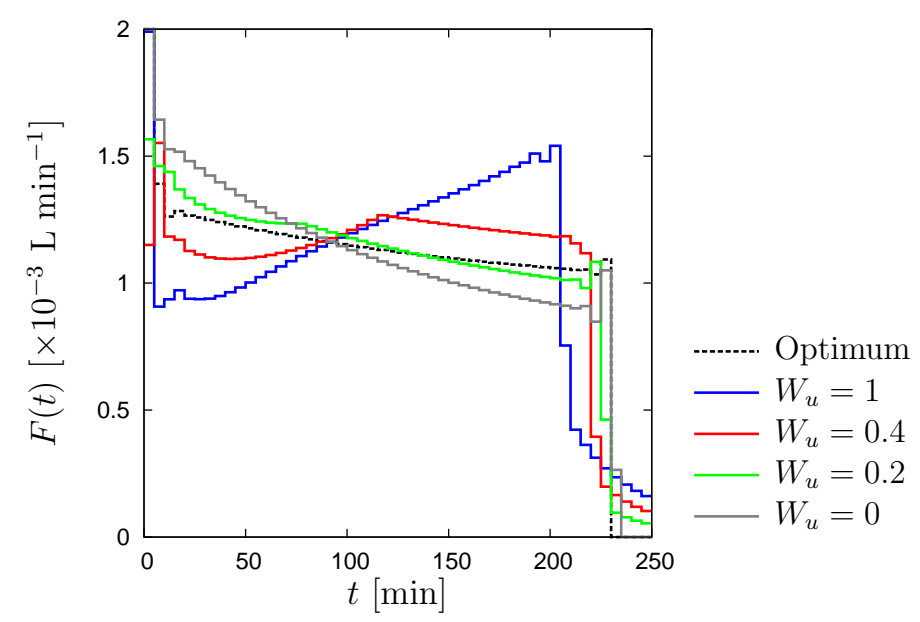

Fig. 10. Compared control profiles (after 20 batches) of integrated two-layer schemes with constraint-filter parameter $W_{\psi}=0.7$ and various input-filter parameters $W_{u}=1.0,0.4,0.2$, and 0 .

Note also that the control stages that are at their bounds at the beginning or the end of the batch vary with the uncertainty. Such changes in the active set of the parameterized optimal control problem thus justify the use of QP approximations, as opposed to a simple linearization of the NCOs, for the NE updates. 


\subsection{Robustness of Integrated Two-Layer Scheme}

The performance of the integrated scheme under noisy conditions is depicted in Figure 11 and compared to constraint adaptation alone, for two scenarios of Gaussian white noise. In turn, the constraint limits are backed-off with twice the amount of measurement noise in order to retain feasibility. Both the integrated two-layer and constraint adaptation schemes exhibit a similar sensitivity to noise in a $1 \%$ standard-deviation scenario (left plot). In contrast, the integrated scheme fails to reduce the effect of noise in a $5 \%$ standard-deviation scenario, compared to constraint adaptation alone (right plot). These results, together with the fact that a full-state measurement is seldom available in practice, are a clear call for use of on-line estimation techniques such as an extended Kalman filter and its unscented variant [33] or a moving horizon estimator [34]. A reduction in noise sensitivity could also be achieved by increasing the frequency of the NE-QP updates.
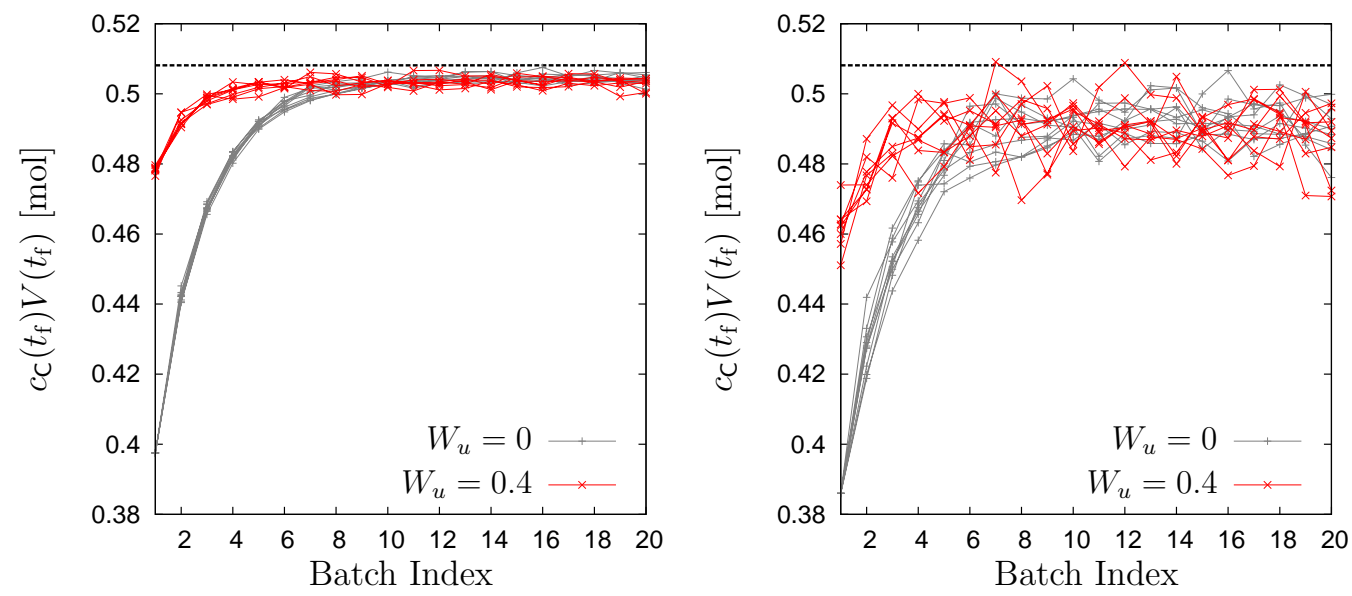

Fig. 11. Compared performance of integrated two-layer scheme $\left(W_{\psi}=0.7\right.$, $\left.W_{u}=0.4\right)$ with constraint-adaptation scheme $\left(W_{\psi}=0.7\right)$ in the presence of Gaussian white noise. Left plot: $1 \%$ standard deviation and $2 \%$ constraint back-offs; Right plots: $5 \%$ standard deviation and $10 \%$ constraint back-offs.

\section{Conclusions}

This paper has considered an integrated two-layer optimization scheme tailored to batch and repetitive processes. The combination of run-to-run constraint adaptation with neighboring-extremal updates is consistent in the sense that the same objective function is used by both layers. Moreover, this combination allows to mitigate the limitations of the two individual schemes. Two variants have been described, namely an indirect and a direct scheme, depending on whether or not the optimal control problem is approximated by a 
finite-dimensional optimization problem. Although less rigorous than the indirect approach, the direct approach can handle singular and path-constrained optimal control problems more readily.

This two-layer architecture is promising in that run-to-run constraint adaptation and neighboring-extremal updates complement each other naturally. The latter brings more reactivity and enhances the convergence speed of the former, in addition to providing a means for rejecting within-run disturbances. Conversely, the integrated scheme inherits the feasibility guarantee of constraint adaptation upon convergence as well as its ability to cope with structural solution changes. These benefits have been demonstrated through the case studies of a connected two-tank system, both in numerical simulation and experimentally, and of a semi-batch reactor, also in numerical simulation.

As part of future work, a theoretical analysis of the improvement in convergence speed of the combined scheme is currently under investigation. Other topics for future research include the ability to correct the gradients of the objective and constraint functions as in the modifier-adaptation approach $[32,53]$, as well as the extension of NE control to perform directional control adaptation [54], in combination with state estimation to enable output feedback.

Acknowledgments The authors gratefully acknowledge the contribution of the Scientific Grant Agency of the Slovak Republic under the grant 1/0053/13 and the Slovak Research and Development Agency under the project APVV0551-11. Part of the work presented in this paper has been completed during a six-month research leave that M. Podmajerský spent in the Department of Chemical Engineering at McMaster University, Canada; this stay was financially supported by the National Scholarship Programme of the Slovak Republic.

\section{References}

[1] J. V. Kadam, W. Marquardt, Integration of economical optimization and control for intentionally transient process operation, Lecture Notes in Control and Information Sciences 358 (2007) 419-434.

[2] F. Allgöwer, A. Zheng, Nonlinear Model Predictive Control, Birkhäuser Verlag, 2000.

[3] C. E. Garcia, D. M. Prett, M. Morari, Model Predictive Control: Theory and Practice - A Survey, Automatica 25 (3) (1989) 335-348.

[4] E. F. Camacho, C. Bordons, Model Predictive Control, Springer-Verlag, London, 1999. 
[5] J. B. Rawlings, R. Amrit, Optimizing process economic performance using model predictive control, Lecture Notes in Control and Information Sciences 384 (2009) 119-138.

[6] V. M. Zavala, C. D. Laird, L. T. Biegler, Fast implementations and rigorous models: Can both be accommodated in NMPC?, International Journal of Robust and Nonlinear Control 18 (2008) 800-815.

[7] M. Diehl, H. G. Bock, J. P. Schlöder, R. Findeisen, Z. Nagy, F. Allgöwer, Real-time optimization and nonlinear model predictive control of processes governed by differential-algebraic equations, Journal of Process Control 12 (4) (2002) 577 - 585.

[8] M. Diehl, J. Gerhard, W. Marquardt, M. Mönnigmann, Numerical solution approaches for robust nonlinear optimal control problems, Computers \& Chemical Engineering 32 (2008) 1279-1292.

[9] L. Würth, R. Hannemann, W. Marquardt, Neighboring-extremal updates for nonlinear model-predictive control and dynamic real-time optimization, Journal of Process Control 19 (8) (2009) 1277-1288.

[10] V. M. Zavala, L. T. Biegler, The advanced-step NMPC controller: Optimality, stability and robustness, Automatica 45 (1) (2009) 86 - 93.

[11] C.-M. Ying, B. Joseph, Performance and stability analysis of LP-MPC and QP-MPC cascade control systems, AIChE Journal 45 (7) (1999) $1521-1534$.

[12] A. Bemporad, M. Morari, V. Dua, E. N. Pistikopoulos, The explicit linear quadratic regulator for constrained systems, Automatica 38 (1) (2002) 320.

[13] E. Pistikopoulos, M. Georgiadis, V. Dua, Multi-Parametric Programming, volume 1 of Process Systems Engineering, Wiley-VCH Verlag, 2007.

[14] E. Pistikopoulos, M. Georgiadis, V. Dua, Multi-Parametric Model-Based Control, volume 2 of Process Systems Engineering, Wiley-VCH Verlag, 2007.

[15] M. Kvasnica, Real-Time Model Predictive Control via Multi-Parametric Programming: Theory and Tools, VDM Verlag, Saarbruecken, 2009.

[16] L. F. Domínguez, D. A. Narciso, E. N. Pistikopoulos, Recent advances in multiparametric nonlinear programming, Computers \& Chemical Engineering 34 (5) (2010) 707-716.

[17] E. N. Pistikopoulos, From multi-parametric programming theory to MPC-on-a-chip multi-scale systems applications, Computers \& Chemical Engineering 47 (2012) 57-66.

[18] B. Srinivasan, D. Bonvin, Real-time optimization of batch processes via tracking of necessary conditions of optimality, Industrial \& Engineering Chemistry Research 46 (2) (2007) 492-504.

[19] J. Kadam, M. Schlegel, B. Srinivasan, D. Bonvin, W. Marquardt, Dynamic optimization in the presence of uncertainty: From off-line nominal solution to measurement-based implementation, Journal of Process Control 17 (2007) 389-398.

[20] S. Gros, B. Chachuat, D. Bonvin, NCO tracking for singular control prob- 
lems using neighboring extremals, in: Proceedings of the 17th IFAC World Congress, Seoul, Korea, 2008, pp. 1922-1927.

[21] D. Bonvin, B. Srinivasan, D. Hunkeler, Control and optimization of batch processes: Improvement of process operation in the production of specialty chemicals, IEEE Control Systems Magazine 26 (6) (2006) 34-45.

[22] A. Marchetti, B. Chachuat, D. Bonvin, Batch process optimization via run-to-run constraints adaptation, in: European Control Conference, Kos, Greece, 2007.

[23] A. E. Bryson, Y.-C. Ho, Applied Optimal Control - Optimization, Estimation and Control, Hemisphere publishing corporation, 1975.

[24] A. V. Fiacco, Introduction to Sensitivity and Stability Analysis in Nonlinear Programming, Vol. 165 of Mathematics in Science and Engineering, Academic Press, New York, 1983.

[25] S. Gros, Neighboring Extremals in Optimization and Control, Ph.D. thesis, Ecole Polytechnique Fédérale de Lausanne (2007).

[26] L. Würth, R. Hannemann, W. Marquardt, A two-layer architecture for economically optimal process control and operation, Journal of Process Control 21 (2011) 311-321.

[27] J. T. Betts, Practical Methods for Optimal Control Using Nonlinear Programming, Advances in Design and Control, SIAM, Philadelphia, 2001.

[28] L. T. Biegler, Nonlinear Programming: Concepts, Algorithms, and Applications to Chemical Processes, MOS-SIAM Series on Optimization, Philadelphia, 2010.

[29] D. E. Kirk, Optimal Control Theory: An Introduction, Prentice-Hall, London, 1970.

[30] S. Subchan, R. Zbikowski, Computational Optimal Control: Tools and Practice, John Wiley \& Sons, Chichester, 2009.

[31] B. Chachuat, A. Marchetti, D. Bonvin, Process optimization via constraints adaptation, Journal of Process Control 18 (2008) 244-257.

[32] B. Chachuat, B. Srinivasan, D. Bonvin, Adaptation strategies for realtime optimization, Computers \& Chemical Engineering 33 (2009) 15571567.

[33] S. Haykin, Kalman Filtering and Neural Networks, John Wiley \& Sons, New York, 2001.

[34] C. Rao, J. Rawlings, Nonlinear moving horizon estimation, in: F. Allgöwer, A. Zheng (Eds.), Nonlinear Model Predictive Control, Vol. 26 of Progress in Systems and Control Theory, Birkhäuser, Basel, 2000, pp. 45-69.

[35] V. M. Zavala, C. D. Laird, L. T. Biegler, A fast moving horizon estimation algorithm based on nonlinear programming sensitivity, Journal of Process Control 18 (2008) 876-884.

[36] S. Gros, B. Srinivasan, D. Bonvin, Optimizing control based on output feedback, Computers \& Chemical Engineering 33 (1) (2009) 191-198.

[37] L. S. Pontryagin, V. G. Boltyanskii, R. V. Gamkrelidze, E. F. Mishchenko, The Mathematical Theory of Optimal Processes, Pergamon Press, New 
York, 1964.

[38] S. Gros, B. Srinivasan, B. Chachuat, D. Bonvin, Neighboring-extremal control for singular dynamic optimization problems. I - Single-input systems, International Journal of Control 82 (6) (2009) 1099-1112.

[39] H. Maurer, H. J. Pesch, Solution differentiability for parametric nonlinear control problems with control-state constraints, Journal of Optimizaton Theory \& Applications 86 (2) (1995) 285-309.

[40] H. Maurer, D. Augustin, Sensitivity analysis and real-time control of parametric optimal control problems using boundary value methods, in: M. Grötschel, S. Krumke, J. Rambau (Eds.), Online Optimization of Large Scale Systems, Springer-Verlag, Berlin, 2001, pp. 17-55.

[41] S. Gros, B. Srinivasan, B. Chachuat, D. Bonvin, Neighboring-extremal control for singular dynamic optimization problems. II - Multiple-input systems, International Journal of Control 82 (7) (2009) 1193-1211.

[42] H. J. Pesch, Real-time computation of feedback controls for constrained optimal control problems. Part I: Neighboring extremals, Optimal Control Applications \& Methods 10 (1989) 129-145.

[43] H. J. Pesch, Real-time computation of feedback controls for constrained optimal control problems. Part II: A correction method based on multiple shooting, Optimal Control Applications \& Methods 10 (1989) 147-171.

[44] T. F. Edgar, D. M. Himmelblau, Optimization of Chemical Processes, McGraw-Hill, New York, 1988.

[45] M. Schlegel, K. Stockmann, T. Binder, W. Marquardt, Dynamic optimization using adaptive control vector parameterization, Computers \& Chemical Engineering 29 (8) (2005) 1731-1751.

[46] H. Pirnay, R. López-Negrete, L. T. Biegler, Optimal sensitivity based on IPOPT, Mathematical Programming Computation 4 (4) (2012) 307-331.

[47] N. Ganesh, L. T. Biegler, A reduced hessian strategy for sensitivity analysis of optimal flowsheets, AIChE Journal 33 (1987) 282-296.

[48] J. V. Kadam, W. Marquardt, Sensitivity-based solution updates in closedloop dynamic optimization, in: Proceedings of the DYCOPS 7 Conference, Elsevier, Amsterdam, 2004.

[49] D. B. Özyurt, P. I. Barton, Cheap second order directional derivatives of stiff ODE embedded functionals, SIAM Journal on Scientific Computing 26 (5) (2005) 1725-1743.

[50] R. Hannemann, W. Marquardt, Continuous and discrete composite adjoints for the hessian of the Lagrangian in shooting algorithms for dynamic optimization, SIAM Journal on Scientific Computing 31 (6) (2010) 4675-4695.

[51] D. Ruppen, D. Bonvin, D. W. T. Rippin, Implementation of adaptive optimal operation for a semi-batch reactor system, Computers \& Chemical Engineering 22 (1998) 185-189.

[52] C. Guntern, A. Keller, K. Hungerbuhler, Economic optimization of an industrial semi-batch reactor applying dynamic programming, Industrial \& Engineering Chemistry Research 37 (10) (1998) 4017-4022. 
[53] A. G. Marchetti, B. Chachuat, D. Bonvin, Modifier-adaptation methodology for real-time optimization, Industrial \& Engineering Chemistry Research 48 (2009) 6022-6033.

[54] S. Deshpande, D. Bonvin, B. Chachuat, Directional input adaptation in parametric optimal control problems, SIAM Journal on Control and Optimization 50 (4) (2012) 1995-2024.

\section{A Derivation of NE Controller for Indirect Scheme}

The following derivation relies on the assumptions stated in Sect. 4. A firstorder approximation of the variations $\delta \boldsymbol{u}(t)$ in the optimal control caused by small changes $\delta \boldsymbol{x}_{i}$ in the state $\boldsymbol{x}\left(t_{i}\right)$ of the dynamic system at a time $t_{i} \in\left[0, t_{\mathrm{f}}\right]$ can be obtained by linearizing the first-order NCOs (8) around the extremal path $\boldsymbol{u}_{k}^{*}$ [23]. Specifically, along each arc composing $\boldsymbol{u}^{*}$, a control variable $u_{i}^{*}(t)$ can either:

- belong to the interior of the control region $u_{i}^{L}<u_{i}^{*}(t)<u_{i}^{U}$, in which case a neighboring-extremal solution is such that $\delta \mu_{i}^{L}(t)=\delta \mu_{i}^{U}(t)=0$, and $\delta u_{i}(t)$ is obtained as

$$
\mathcal{H}_{u_{i} \boldsymbol{x}}^{*} \delta \boldsymbol{x}(t)+\boldsymbol{F}_{u_{i}}^{* T} \delta \boldsymbol{\lambda}(t)+\mathcal{H}_{\boldsymbol{u} u_{i}}^{*} \delta u_{i}(t)=\mathbf{0}
$$

- or be at one of its boundaries $u_{i}^{L}$ or $u_{i}^{U}$, in which case a NE control is simply given by $\delta u_{i}(t)=0$.

These $n_{u}$ conditions can be written collectively in the form

$$
\boldsymbol{A}^{*}(t) \delta \boldsymbol{\lambda}(t)+\boldsymbol{B}^{*}(t) \delta \boldsymbol{x}+\boldsymbol{C}^{*}(t) \delta \boldsymbol{u}(t)=\mathbf{0},
$$

where $\boldsymbol{A}^{*}(t), \boldsymbol{B}^{*}(t) \in \mathbb{R}^{n_{u} \times n_{x}}, \boldsymbol{C}^{*}(t) \in \mathbb{R}^{n_{u} \times n_{u}}$. Moreover, the linearization of the two-point boundary value problem (TPBVP) in the variables $\left(\boldsymbol{x}^{*}, \boldsymbol{\lambda}^{*}\right)$ gives

$$
\begin{aligned}
\delta \dot{\boldsymbol{x}}(t) & =\boldsymbol{F}_{\boldsymbol{x}}^{*} \delta \boldsymbol{x}(t)+\boldsymbol{F}_{\boldsymbol{u}}^{*} \delta \boldsymbol{u}(t) \\
\delta \dot{\boldsymbol{\lambda}}(t) & =-\boldsymbol{F}_{\boldsymbol{x}}^{* T} \delta \boldsymbol{\lambda}(t)-\mathcal{H}_{\boldsymbol{x} \boldsymbol{x}}^{*} \delta \boldsymbol{x}(t)-\mathcal{H}_{\boldsymbol{x} \boldsymbol{u}}^{*} \delta \boldsymbol{u}(t)
\end{aligned}
$$

with the linearized boundary conditions

$$
\begin{aligned}
& \delta \boldsymbol{x}\left(t_{i}\right)=\delta \boldsymbol{x}_{i} \\
& \delta \boldsymbol{\lambda}\left(t_{\mathrm{f}}\right)=\left[\boldsymbol{\Phi}_{\boldsymbol{x} \boldsymbol{x}}^{*}\right]_{t_{\mathrm{f}}} \delta \boldsymbol{x}\left(t_{\mathrm{f}}\right)+\left[\overline{\boldsymbol{\psi}}_{\boldsymbol{x}}^{*}\right]_{t_{\mathrm{f}}} \delta \overline{\boldsymbol{\nu}}^{*} \\
& \mathbf{0}=\left[\overline{\boldsymbol{\psi}}_{\boldsymbol{x}}^{*}\right]_{t_{\mathrm{f}}} \delta \boldsymbol{x}\left(t_{\mathrm{f}}\right) .
\end{aligned}
$$


If the second-order sufficiency conditions (SOSCs) in $[39,40]$ hold, the optimal control problem (1)-(4)\&(6) is regular. Therefore, $\boldsymbol{C}^{*}$ is invertible along $0 \leq$ $t \leq t_{\mathrm{f}}$, and a NE control law readily obtained in the form

$$
\delta \boldsymbol{u}(t)=-\boldsymbol{C}^{*}(t)^{-1}\left[\boldsymbol{A}^{*}(t) \delta \boldsymbol{\lambda}(t)+\boldsymbol{B}^{*}(t) \delta \boldsymbol{x}(t)\right] .
$$

Note that this control law reduces to the standard NE control law in the case where no input constraint is active; that is

$$
\boldsymbol{A}^{*}=\boldsymbol{F}_{\boldsymbol{u}}^{* \top}, \quad \boldsymbol{B}^{*}=\mathcal{H}_{u x}^{*}, \quad \boldsymbol{C}^{*}=\mathcal{H}_{u \boldsymbol{u}}^{*} .
$$

Overall, $\delta \boldsymbol{x}(t)$ and $\delta \boldsymbol{\lambda}(t)$ satisfy the following linear TPBVP:

$$
\begin{aligned}
& \left(\begin{array}{l}
\delta \dot{\boldsymbol{x}}(t) \\
\delta \dot{\boldsymbol{\lambda}}(t)
\end{array}\right)=\boldsymbol{\Delta}(t)\left(\begin{array}{c}
\delta \boldsymbol{x}(t) \\
\delta \boldsymbol{\lambda}(t)
\end{array}\right), \\
& \delta \boldsymbol{x}(0)=\delta \boldsymbol{x}_{0}, \quad \delta \overline{\boldsymbol{\psi}}=\left[\overline{\boldsymbol{\psi}}_{\boldsymbol{x}}^{*} \delta \boldsymbol{x}\right]_{t=t_{\mathrm{f}}}, \\
& \delta \boldsymbol{\lambda}\left(t_{\mathrm{f}}\right)=\left[\left(\boldsymbol{\phi}_{\boldsymbol{x} \boldsymbol{x}}^{*}+\overline{\boldsymbol{\nu}}^{* T} \overline{\boldsymbol{\psi}}_{\boldsymbol{x} \boldsymbol{x}}^{*}\right) \delta \boldsymbol{x}+\overline{\boldsymbol{\psi}}_{\boldsymbol{x}}^{* T} \delta \overline{\boldsymbol{\nu}}\right]_{t=t_{\mathrm{f}}}
\end{aligned}
$$

with

$$
\boldsymbol{\Delta}(t)=\left(\begin{array}{cc}
\boldsymbol{F}_{\boldsymbol{x}}^{*}-\boldsymbol{F}_{u}^{*} \boldsymbol{C}^{*}(t)^{-1} \boldsymbol{B}^{*}(t) & -\boldsymbol{F}_{u}^{*} \boldsymbol{C}^{*}(t)^{-1} \boldsymbol{A}^{*}(t) \\
-\mathcal{H}_{\boldsymbol{x} \boldsymbol{x}}^{*}+\mathcal{H}_{\boldsymbol{x} \boldsymbol{u}}^{*} \boldsymbol{C}^{*}(t)^{-1} \boldsymbol{B}^{*}(t) & -\boldsymbol{F}_{\boldsymbol{x}}^{* T}+\mathcal{H}_{\boldsymbol{x} \boldsymbol{u}}^{*} \boldsymbol{C}^{*}(t)^{-1} \boldsymbol{A}^{*}(t)
\end{array}\right) .
$$

Also under the SOSCs in $[39,40]$, the control value along the interior arc and the control bound are equal at a switching time $t_{k}$ between a lower or upper bound and an interior arc. It follows that $u_{i}^{\mathcal{H}}\left(t_{k}\right)=u_{i}^{L}$ or $u_{i}^{\mathcal{H}}\left(t_{k}\right)=u_{i}^{U}$, where $u_{i}^{\mathcal{H}}$ represents the control obtained from solving the condition $\mathcal{H}_{u_{i}}=0$. In addition, the state and adjoint trajectories are themselves continuous at $t_{k}$ :

$$
\boldsymbol{x}^{*}\left(t_{k}^{+}\right)=\boldsymbol{x}^{*}\left(t_{k}^{-}\right), \quad \boldsymbol{\lambda}^{*}\left(t_{k}^{+}\right)=\boldsymbol{\lambda}^{*}\left(t_{k}^{-}\right)
$$

These additional conditions provides a means of computing the variations in switching times. For simplicity, however, the NE controller used in the inner layer of the two-layer optimization scheme considers fixed switching; it is in the outer layer of the integrated scheme that accounts for these variations. 